\title{
The asymptotic of curvature of direct image bundle associated with higher powers of a relatively ample line bundle
}

\section{Xueyuan Wan ${ }^{1} \cdot$ Genkai Zhang ${ }^{2}$ (D)}

Received: 12 June 2020 / Accepted: 21 April 2021 / Published online: 11 May 2021

(C) The Author(s) 2021

\begin{abstract}
Let $\pi: \mathcal{X} \rightarrow M$ be a holomorphic fibration with compact fibers and $L$ a relatively ample line bundle over $\mathcal{X}$. We obtain the asymptotic of the curvature of $L^{2}$-metric and Qullien metric on the direct image bundle $\pi_{*}\left(L^{k} \otimes K_{\mathcal{X} / M}\right)$ up to the lower order terms than $k^{n-1}$, for large $k$. As an application we prove that the analytic torsion $\tau_{k}(\bar{\partial})$ satisfies $\partial \bar{\partial} \log \left(\tau_{k}(\bar{\partial})\right)^{2}=o\left(k^{n-1}\right)$, where $n$ is the dimension of fibers.
\end{abstract}

Keywords Holomorphic fibration · Quillen metric $\cdot L^{2}$-metric $\cdot$ Curvature $\cdot$ Asymptotics

Mathematics Subject Classification $32 \mathrm{G} 08 \cdot$ 53C55

\section{Introduction}

Let $\pi: \mathcal{X} \rightarrow M$ be a holomorphic fibration with compact fibers and $L$ a relatively ample line bundle over $\mathcal{X}$, i.e. there is a smooth metric (weight) $\phi$ on $L$ such that the first Chern form $\frac{\sqrt{-1}}{2 \pi} \partial \bar{\partial} \phi$ is positive $(1,1)$-form along each fiber. One may consider the following direct image bundle

$$
E^{k}=\pi_{*}\left(L^{k} \otimes K_{\mathcal{X} / M}\right) .
$$

Here $K_{\mathcal{X} / M}=K_{\mathcal{X}} \otimes \pi^{*} K_{M}^{-1}$ denotes the relative canonical line bundle. The bundle $E^{k}$ is equipped with the canonical $L^{2}$-metric

$$
\|u\|^{2}:=\int_{\mathcal{X}_{y}}|u|^{2} e^{-\phi}, \quad u \in E_{y}^{k}, \quad y \in M ;
$$

\section{Genkai Zhang}

genkai@chalmers.se

Xueyuan Wan

xwan@cqut.edu.cn

1 Mathematical Science Research Center, Chongqing University of Technology, Chongqing 400054, China

2 Mathematical Sciences, Chalmers University of Technology, 41296 Gothenburg, Sweden 
see [6-8]. Here $|u|^{2} e^{-\phi}$ is defined as follows: $u$ can be written locally as $u=u^{\prime} d v \wedge e$, where $e$ is a local holomorphic frame for $\left.L\right|_{\mathcal{X}_{y}}, \mathcal{X}_{y}=\pi^{-1}(y)$, and

$$
|u|^{2} e^{-\phi}:=(\sqrt{-1})^{n^{2}}\left|u^{\prime}\right|^{2}|e|^{2} d v \wedge d \bar{v}=(\sqrt{-1})^{n^{2}}\left|u^{\prime}\right|^{2} e^{-\phi} d v \wedge d \bar{v},
$$

where $d v=d v^{1} \wedge \cdots \wedge d v^{n}$.

In [8, Theorem 1.2] Berndtsson computed the curvature $\Theta^{E_{k}}$ of the $L^{2}$-metric and consequently proved Nagano positivity. More precisely,

$$
\left\langle\sqrt{-1} \Theta^{E_{k}} u, u\right\rangle=\int_{\mathcal{X} / M} k c(\phi)|u|^{2} e^{-k \phi}+k\left\langle\left(\Delta^{\prime}+k\right)^{-1} i_{\mu_{\alpha}} u, i_{\mu_{\beta}} u\right\rangle \sqrt{-1} d z^{\alpha} \wedge d \bar{z}^{\beta},
$$

where the definitions of $c(\phi), \mu_{\alpha}$ and $\Delta^{\prime}$ are given in Theorem 2.2.

In particular, if $\pi: \mathcal{X} \rightarrow M$ is a trivial fibration, Berndtsson [7, Theorem 4.1, 4.2] obtained an asymptotic of $\operatorname{tr} \Theta^{E_{k}} / d_{k}$ up to $o(1), d_{k}=\operatorname{rank} E_{k}$. In view of the relation of the $L^{2}$-curvature with analytic torsion and Quillen metric it is a natural and interesting problem to find the lower order terms in the asymptotic of $\operatorname{tr} \Theta^{E_{k}}$ for a general fibration. We solve the problem up to reminder term of order $o\left(k^{n-1}\right)$.

Theorem 1.1 For any vector $\zeta \in T_{y} M$, we have

$$
\begin{aligned}
- & \sqrt{-1} c_{1}\left(E^{k},\|\bullet\|_{k}\right)(\zeta, \bar{\zeta}) \\
= & \frac{k^{n+1}}{(2 \pi)^{n+1}} \int_{\mathcal{X}_{y}}(-\sqrt{-1}) c(\phi)(\zeta, \bar{\zeta}) \frac{\omega^{n}}{n !}+\frac{k^{n}}{(2 \pi)^{n+1}} \int_{\mathcal{X}_{y}}\left(\frac{1}{2}|\mu|^{2}-\frac{\rho}{2}(-\sqrt{-1}) c(\phi)(\zeta, \bar{\zeta})\right) \frac{\omega^{n}}{n !} \\
& +\frac{k^{n-1}}{(2 \pi)^{n+1}} \int_{\mathcal{X}_{y}}\left((-\sqrt{-1}) c(\phi)(\zeta, \bar{\zeta})\left(-\frac{1}{6} \Delta \rho+\frac{1}{24}\left(|R|^{2}-4|R i c|^{2}+3 \rho^{2}\right)\right)-\frac{\rho}{4}|\mu|^{2}\right) \frac{\omega^{n}}{n !} \\
& +\frac{k^{n-1}}{(2 \pi)^{n+1}}\left(\frac{1}{12}\|\mu\|_{R i c}^{2}+\frac{1}{12}\left\|\nabla^{\prime} \mu\right\|^{2}-\frac{1}{4}\left\|\bar{\partial}^{*} \mu\right\|^{2}\right)+o\left(k^{n-1}\right) .
\end{aligned}
$$

We refer to the Sect. 3.1 for the definitions and notation.

We note that the first two terms in the expansion above were proved by Ma-Zhang [22]. The leading terms of the first summand in (1.2) is studied by Sun [24] and the second by Berndtsson [7] (in the different setup of trivial fibration with variation of Kähler metrics).

We shall then compare our expansion for the $L^{2}$-curvature with the Quillen curvature. So let $D_{y}=\bar{\partial}_{y}+\bar{\partial}_{y}^{*}$ be the Dirac operator acting on $A^{0, *}\left(\mathcal{X}_{y}, L^{k} \otimes K \mathcal{X} / M\right)$ of $(0, *)$-forms, where $\mathcal{X}_{y}$ is endowed with the restricted Hermitian metric $(\sqrt{-1} \partial \bar{\partial} \phi) \mid \mathcal{X}_{y}$. For any $0<b<c$, denote by $D_{i}^{(b, c)}$ the restriction of $D$ on the sum of eigenspaces of $A^{0, i}\left(\mathcal{X}_{y}, L^{k} \otimes K \mathcal{X} / M\right)$ for eigenvalues in $(b, c), K_{\mathcal{X} / M}$ being equipped a natural Hermitian metic

$$
(\operatorname{det} \phi)^{-1}:=\left(\operatorname{det}\left(\left.\partial \bar{\partial} \phi\right|_{\mathcal{X}_{y}}\right)\right)^{-1} .
$$

Then the (Ray-Singer) analytic torsion is defined by

$$
\begin{aligned}
\tau_{k}(\bar{\partial}) & =\tau_{k}\left(\bar{\partial}^{(b,+\infty)}\right) \\
& =\left(\left(\operatorname{det}\left(D_{1}^{(b,+\infty)}\right)^{2}\right)\left(\operatorname{det}\left(D_{2}^{(b,+\infty)}\right)^{2}\right)^{-2}\left(\operatorname{det}\left(D_{3}^{(b,+\infty)}\right)^{2}\right)^{3} \ldots\right)^{1 / 2},
\end{aligned}
$$

and is a positive smooth function on the base manifold $M$. Here $b$ is a constant less than all positive eigenvalues of $D$ (see Definition 2.6). 
The Quillen metric $\|\bullet\|_{Q}$ on the determinant line $\lambda$ (see Definition 2.5) is patched by the $L^{2}$-metric $|\bullet|^{b}$ on $\lambda^{b}($ see $(2.13))$ and the analytic torsion $\tau_{k}(\bar{\partial})$, i.e.

$$
\|\bullet\|_{Q}=|\bullet|{ }^{b} \tau_{k}(\bar{\partial}),
$$

where $b>0$ is a sufficiently small constant.

In their papers [11-13], J.-M. Bismut, H. Gillet and C. Soulé computed the curvature of Quillen metric for a locally Kähler family and obtained the differential form version of Grothendieck-Riemann-Roch Theorem. More precisely, they proved that as holomorphic bundles,

$$
\lambda_{y} \cong \bigotimes_{i \geq 0} \operatorname{det} H^{i}\left(\mathcal{X}_{y}, L^{k} \otimes K_{\mathcal{X} / M}\right)^{(-1)^{i+1}}
$$

and the curvature is

$$
c_{1}\left(\lambda,\|\bullet\|_{Q}\right)=-\left\{\int_{\mathcal{X} / M} \operatorname{Td}\left(\frac{-R^{T \mathcal{X} / M}}{2 \pi i}\right) \operatorname{Tr}\left[\exp \left(\frac{-R^{L^{k} \otimes K_{\mathcal{X} / M}}}{2 \pi i}\right)\right]\right\}^{(1,1)} .
$$

Since $L$ is a relatively ample line bundle over $\mathcal{X}$, by Kodaira vanishing theorem,

$$
H^{i}\left(\mathcal{X}_{y}, K_{\mathcal{X} / M} \otimes L^{k}\right)=0
$$

for all $i \geq 1$. Therefore,

$$
\lambda \cong\left(\operatorname{det} E^{k}\right)^{-1} .
$$

We expand also the Quillen curvature $c_{1}\left(\lambda,\|\bullet\|_{Q}\right)$ and compare it with (1.3). We prove

Theorem 1.2 Up to terms of order $o\left(k^{n-1}\right)$ the Quillen curvature $-c_{1}(\lambda,\|\bullet\| Q)$ has the same expansion (1.3) as for the $L^{2}$-curvature.

As application we shall find the asymptotics of the variation of the analytic torsion. From (3.56) we have

$$
\operatorname{det}\|\bullet\|_{k}^{2}=\left(\left(|\bullet|^{b}\right)^{2}\right)^{*}
$$

for $b>0$ a sufficiently small constant, where det $\|\bullet\|_{k}$ denotes the natural induced $L^{2}$-metric on line bundle $\operatorname{det} E^{k}$ and $\left(\left(|\bullet|^{b}\right)^{2}\right)^{*}$ denotes the dual metric of $\left(|\bullet|^{b}\right)^{2}$. Using (1.4) and (1.7) we have furthermore

$$
\frac{\sqrt{-1}}{2 \pi} \partial \bar{\partial} \log \left(\tau_{k}(\bar{\partial})\right)^{2}=-c_{1}\left(\lambda,\|\bullet\|_{Q}\right)-c_{1}\left(E^{k},\|\bullet\|_{k}\right) .
$$

As an immediate consequence of Theorem 1.1 and Theorem 1.2 we have

Corollary 1.3 As $k \rightarrow \infty$, we have

$$
\partial \bar{\partial} \log \left(\tau_{k}(\bar{\partial})\right)^{2}=o\left(k^{n-1}\right) .
$$

Here the asymptotic (1.9) is understood as $\left(\partial \bar{\partial} \log \left(\tau_{k}(\bar{\partial})\right)^{2}\right)(\zeta, \bar{\zeta})=o\left(k^{n-1}\right)$ for any vector $\zeta \in T M$.

Remark 1.4 The asymptotic of analytic torsion has been studied by $[5,10]$. It is proved in [10, Theorem 8] the coefficients of $k^{n}, k^{n} \log k$ are topological invariants. After a preliminary version of this paper was finished, we were informed by Xiaonan Ma of the paper [19, Theorem 1.1, 1.2] by Finski where the coefficients of $k^{n-1}, k^{n-1} \log k$ in the analytic torsion $\tau_{k}$ have also been computed, which implies then (1.9). However, our method here is completely different from the methods in $[10,19]$. 
Remark 1.5 For the case of $M$ is the Teichmüller space of compact Riemann surfaces of genus $g \geq 2$ and $L$ is the relative canonical line bundle, Corollary 1.3 was proved in [18]; in this case the analytic torsion $\tau_{k}$ actually decays exponentially in $k$.

We proceed to explain briefly our method for the expansion in Theorem 1.1. By the formula (1.2), the first Chern form $c_{1}\left(E^{k},\|\bullet\|_{k}\right)$ is the trace of an integral operator, and in the paper [22] X. Ma and W. Zhang found the expansion of the diagonal of the kernel of the operators, proving a local index formula. To find the third order term, i.e. the coefficient of $k^{n-1}$, seems a difficult task and requires much more effort. The trace of first summand in (1.2) is relatively easy to handle, however the second summand involves Toeplitz operators with symbols being differential operators. A major ingredient of our method is the following expansion (see Lemma 3.1),

$$
\begin{aligned}
\left(\Delta^{\prime}+k\right)^{-1}= & \frac{1}{2 k}+\frac{1}{6 k^{2}}\left(k-\Delta^{\prime}-R^{*}\right)+\frac{1}{4 k^{2}} R^{*}+\frac{1}{4 k^{2}}\left(\Delta^{\prime}+k\right)^{-1}\left(k-\Delta^{\prime}\right) R^{*} \\
& +\frac{1}{18 k^{3}}\left(2 k-\Delta^{\prime}\right)\left(k-\Delta^{\prime}-R^{*}\right)+\frac{1}{36 k^{4}}\left(2 k-\Delta^{\prime}\right)^{2}\left(k-\Delta^{\prime}-R^{*}\right) \\
& +\frac{1}{36 k^{4}}\left(\Delta^{\prime}+k\right)^{-1}\left(k-\Delta^{\prime}\right)\left(2 k-\Delta^{\prime}\right)^{2}\left(k-\Delta^{\prime}-R^{*}\right)
\end{aligned}
$$

where $R^{*}=R_{i \bar{l}}^{\bar{t} j} i_{\frac{\partial}{\partial v^{j}}} d v^{i} \wedge d \bar{v}^{l} \wedge i_{\frac{\partial}{\partial \bar{v}^{t}}}, R_{i \bar{l}}^{\bar{t} j}=-\partial_{i} \partial_{\bar{l}} \phi^{\bar{t} j}+\phi_{\bar{l}}^{\bar{t} s} \phi_{i}^{\bar{k} j} \phi_{s \bar{k}}$ is the Chern curvature component of $\left(T_{X}^{*},\left(\phi^{i} \bar{j}\right)\right)$. The corresponding contribution of each term above to the $L^{2}$ curvature $c_{1}\left(E^{k},\|\bullet\|_{k}\right)$ will be effectively treated by using further the asymptotic expansion of Bergman Kernel for bundles [26, Theorem 4.2].

We note that generally it is always interesting to study variations of complex or Kähler structures and connections on bundles of cohomology spaces over moduli spaces. The most well-known case might be the Siegel moduli space parametrising polarized Abelian varieties. This aspect has been very much studied in mathematical physics; see e.g. [1-3] and references therein.

This article is organized as follows. In Sect. 2, we fix notation and recall some basic facts on Berndtsson's curvature formula of $L^{2}$-metric, the asymptotic expansion of Bergman kernel for bundles, analytic torsion and Quillen metric. In Sect. 3, we find the expansion of $c_{1}\left(E^{k},\|\bullet\|_{k}\right)$ and prove Theorem 1.1. We also give the expansion of $-c_{1}\left(\lambda,\|\bullet\|_{Q}\right)$ and prove Theorem 1.2. By comparing with their expansions, we prove Corollary 1.3.

\section{Preliminaries}

We shall fix notation and recall some necessary background material.

\subsection{Berndtsson's curvature formula of $L^{2}$-metric}

We refer [6-8] and references therein.

Let $\pi: \mathcal{X} \rightarrow M$ be a holomorphic fibration with compact fibres and $L$ a relatively ample line bundle over $\mathcal{X}$. We denote by $(z ; v)=\left(z^{1}, \ldots, z^{m} ; v^{1}, \ldots, v^{n}\right)$ a local admissible holomorphic coordinate system of $\mathcal{X}$ with $\pi(z ; v)=z$, where $m=\operatorname{dim}_{\mathbb{C}} M, n=\operatorname{dim}_{\mathbb{C}} \mathcal{X}-$ $\operatorname{dim}_{\mathbb{C}} M$. 
For any smooth function $\phi$ on $\mathcal{X}$, we denote

$$
\phi_{\alpha}:=\frac{\partial \phi}{\partial z^{\alpha}}, \quad \phi_{\bar{\beta}}:=\frac{\partial \phi}{\partial \bar{z}^{\beta}}, \quad \phi_{i}:=\frac{\partial \phi}{\partial v^{i}}, \quad \phi_{\bar{j}}:=\frac{\partial \phi}{\partial \bar{v}^{j}},
$$

where $1 \leq i, j \leq n, 1 \leq \alpha, \beta \leq m$.

Let $F^{+}(L)$ be the space of smooth metrics $\phi$ on $L$ with

$$
\left.(\sqrt{-1} \partial \bar{\partial} \phi)\right|_{\mathcal{X}_{y}}>0
$$

for any point $y \in M$. For any $\phi \in F^{+}(L)$, set

$$
\frac{\delta}{\delta z^{\alpha}}:=\frac{\partial}{\partial z^{\alpha}}-\phi_{\alpha \bar{j}} \phi^{\bar{j} k} \frac{\partial}{\partial v^{k}} .
$$

By a routine computation, one can show that $\left\{\frac{\delta}{\delta z^{\alpha}}\right\}_{1 \leq \alpha \leq m}$ spans a well-defined horizontal subbundle of $T \mathcal{X}$.

Let $\left\{d z^{\alpha} ; \delta v^{k}\right\}$ denote the dual frame of $\left\{\frac{\delta}{\delta z^{\alpha}} ; \frac{\partial}{\partial v^{i}}\right\}$. Then

$$
\delta v^{k}=d v^{k}+\phi^{k \bar{l}} \phi_{\bar{l} \alpha} d z^{\alpha}
$$

Moreover, the differential operators

$$
\partial^{V}=\frac{\partial}{\partial v^{i}} \otimes \delta v^{i}, \quad \partial^{H}=\frac{\delta}{\delta z^{\alpha}} \otimes d z^{\alpha} .
$$

are well-defined.

For any $\phi \in F^{+}(L)$, the geodesic curvature $c(\phi)$ is defined by

$$
c(\phi)=\left(\phi_{\alpha \bar{\beta}}-\phi_{\alpha \bar{j}} \phi^{i \bar{j}} \phi_{i \bar{\beta}}\right) \sqrt{-1} d z^{\alpha} \wedge d \bar{z}^{\beta},
$$

which is a horizontal real $(1,1)$-form on $\mathcal{X}$. The following lemma confirms that the geodesic curvature $c(\phi)$ of $\phi$ is indeed well-defined.

Lemma 2.1 [17] The following decomposition holds,

$$
\sqrt{-1} \partial \bar{\partial} \phi=c(\phi)+\sqrt{-1} \phi_{i} \bar{j} \delta v^{i} \wedge \delta \bar{v}^{j} .
$$

Proof This is a direct computation,

$$
\begin{aligned}
c(\phi) & +\sqrt{-1} \phi_{i \bar{j}} \delta v^{i} \wedge \delta \bar{v}^{j}=\sqrt{-1}\left(\phi_{\alpha \bar{\beta}}-\phi_{\alpha \bar{l}} \phi^{k \bar{l}} \phi_{k \bar{\beta}}\right) d z^{\alpha} \wedge d \bar{z}^{\beta} \\
& +\sqrt{-1} \phi_{i \bar{j}}\left(d v^{i}+\phi^{i \bar{l}} \phi_{\overline{l \alpha}} d z^{\alpha}\right) \wedge\left(d \bar{v}^{j}+\phi^{\bar{j} k} \phi_{k \bar{\beta}} d \bar{z}^{\beta}\right) \\
= & \sqrt{-1}\left(\phi_{\alpha \bar{\beta}} d z^{\alpha} \wedge d \bar{z}^{\beta}+\phi_{\alpha \bar{j}} d z^{\alpha} \wedge d \bar{v}^{j}+\phi_{i \bar{\beta}} d v^{i} \wedge d \bar{z}^{\beta}+\phi_{i \bar{j}} d v^{i} \wedge d \bar{v}^{j}\right) \\
= & \sqrt{-1} \partial \bar{\partial} \phi .
\end{aligned}
$$

Following Berndtsson (cf. [6-8]) we consider the direct image bundle $E:=\pi_{*}\left(K_{\mathcal{X} / M} \otimes\right.$ $L$ ), and define the following $L^{2}$-metric on $E$ : for $y \in M, \mathcal{X}_{y}=\pi^{-1}(y)$, and $u \in E_{y} \equiv$ $H^{0}\left(\mathcal{X}_{y},\left(L \otimes K_{\mathcal{X} / M}\right)_{y}\right)$,

$$
\|u\|^{2}:=\int_{\mathcal{X}_{y}}|u|^{2} e^{-\phi} .
$$


Note that $u$ can be written locally as $u=u^{\prime} d v \wedge e$, where $e$ is a local holomorphic frame for $\left.L\right|_{\mathcal{X}}$, and so locally

$$
|u|^{2} e^{-\phi}=(\sqrt{-1})^{n^{2}}\left|u^{\prime}\right|^{2}|e|^{2} d v \wedge d \bar{v}=(\sqrt{-1})^{n^{2}}\left|u^{\prime}\right|^{2} e^{-\phi} d v \wedge d \bar{v},
$$

where $d v=d v^{1} \wedge \cdots \wedge d v^{n}$ is the fiber volume.

By the definition of $\bar{\partial} V$, we have

$$
\mu_{\alpha}=\bar{\partial}^{V}\left(\frac{\delta}{\delta z^{\alpha}}\right)=-\frac{\partial}{\partial \bar{v}^{l}}\left(\phi_{\alpha \bar{j}} \phi^{\bar{j} i}\right) d \bar{v}^{l} \otimes \frac{\partial}{\partial v^{i}},
$$

which is in the Kodaira-Spencer class $\rho\left(\left.\frac{\partial}{\partial z^{\alpha}}\right|_{y}\right) \in H^{1}\left(\mathcal{X}_{y}, T_{\mathcal{X}_{y}}\right)$.

The following theorem was proved by Berndtsson in [8, Theorem 1.2], its proof also can be found in [17, Theorem 3.1].

Theorem 2.2 [8] For any $y \in M$ the curvature $\left\langle\Theta^{E}(u, u)\right\rangle, u \in E_{y}$, of the Chern connection on $E$ with the $L^{2}$ metric is given by

$$
\left\langle\sqrt{-1} \Theta^{E} u, u\right\rangle=\int_{\mathcal{X}_{y}} c(\phi)|u|^{2} e^{-\phi}+\left\langle\left(1+\Delta^{\prime}\right)^{-1} i_{\mu_{\alpha}} u, i_{\mu_{\beta}} u\right\rangle \sqrt{-1} d z^{\alpha} \wedge d \bar{z}^{\beta} .
$$

Here $\Delta^{\prime}=\nabla^{\prime} \nabla^{*}+\nabla^{*} \nabla$ is the Laplacian on $\left.L\right|_{\mathcal{X}_{y}}$-valued forms on $\mathcal{X}_{y}$ defined by the $(1,0)$-part of the Chern connection on $\left.L\right|_{\mathcal{X}_{y}}$.

We replace now the Hermitian line bundle $\left(L, e^{-\phi}\right)$ by its powers $\left(L^{k}, e^{-k \phi}\right)$, and consider the corresponding direct image bundle $E^{k}:=\pi_{*}\left(L^{k} \otimes K_{\mathcal{X} / M}\right)$. Let $\nabla_{k}^{\prime *}$ (resp. $\left.\nabla^{\prime *}\right)$ be the adjoint operator of $\nabla^{\prime}$ with respect to $\left(L^{k}, e^{-k \phi}\right)$ and $(X, k \omega=k \sqrt{-1} \partial \bar{\partial} \phi)$ (resp. $(X, \omega=\sqrt{-1} \partial \bar{\partial} \phi))$. We have

$$
\nabla^{*}=\sqrt{-1}\left[\Lambda_{k \omega}, \nabla^{\prime}\right]=\frac{1}{k} \sqrt{-1}\left[\Lambda_{\omega}, \nabla^{\prime}\right]=\frac{1}{k} \nabla^{*},
$$

acting on sections of $E_{k}$ with $\nabla^{\prime *}$ corresponding the fixed background metric $(X, \omega=$ $\sqrt{-1} \partial \bar{\partial} \phi)$. It implies that

$$
\Delta_{k}^{\prime}=\nabla_{k}^{*} \nabla^{\prime}+\nabla^{\prime} \nabla_{k}^{*}=\frac{1}{k} \Delta^{\prime}
$$

From Theorem 2.2 and (2.6), the curvature of $L^{2}$-metric (see (2.3)) on $E^{k}$ is given by

$$
\begin{aligned}
\left\langle\sqrt{-1} \Theta^{E^{k}} u, u\right\rangle & =\int_{\mathcal{X}_{y}} c(k \phi)|u|^{2} e^{-k \phi}+\left\langle\left(1+\Delta_{k}^{\prime}\right)^{-1} i_{\mu_{\alpha}} u, i_{\mu_{\beta}} u\right\rangle_{k \omega} \sqrt{-1} d z^{\alpha} \wedge d \bar{z}^{\beta} \\
& =\int_{\mathcal{X}_{y}} k c(\phi)|u|^{2} e^{-k \phi}+k\left\langle\left(k+\Delta^{\prime}\right)^{-1} i_{\mu_{\alpha}} u, i_{\mu_{\beta}} u\right\rangle \sqrt{-1} d z^{\alpha} \wedge d \bar{z}^{\beta}
\end{aligned}
$$

for any element $u$ of $E_{y}^{k}$.

\subsection{The Bergman kernels}

Let $(X, \omega)$ be a compact Kähler manifold of $n$-dimension, $\left(L, e^{-\phi}\right)$ be a Hermitian line bundle over $X$ satisfying

$$
\sqrt{-1} R^{L}=\sqrt{-1} \partial \bar{\partial} \phi=\omega .
$$

We shall eventually replace $(X, \omega)$ by the fibers of the fibration in the previous section. 
Let $(E, H)$ be a Hermitian vector bundle over $X$. There is a natural $L^{2}$-metric on the space $H^{0}\left(X, L^{k} \otimes E\right)$ of holomorphic forms of $L^{k} \otimes E, k \geq 0$. Let $\left\{u_{j}\right\}_{j=1}^{N_{k}}$ be an orthonormal basis of $H^{0}\left(X, L^{k} \otimes E\right)$, where $d_{k}=\operatorname{dim} H^{0}\left(X, L^{k} \otimes E\right)$. The $k$-th Bergman kernel $B_{k}(H) \in$ $\operatorname{End}(E)$ is defined by

$$
B_{k}(H)=\sum_{j=1}^{d_{k}} u_{j}^{*} \otimes u_{j} .
$$

Recall the following Tian-Yau-Zelditch expansion of Bergman kernel for bundles. We use the version in [14] and refer [4,16,20,25-28] for different variations and proofs.

Theorem 2.3 For a fixed metric $H$, there is an asymptotic expansion as $k \rightarrow \infty$,

$$
B_{k}(H)=\frac{1}{(2 \pi)^{n}}\left(A_{0} k^{n}+A_{1} k^{n-1}+\cdots\right),
$$

where $A_{i} \in \operatorname{End}(E)$ are determined by the geometry of $\omega$ and $H$. The expansion is in the sense that for any integer $l, R \geq 0$,

$$
\left\|(2 \pi)^{n} B_{k}(H)-\sum_{j<R} A_{j} k^{n-j}\right\|_{C^{l}} \leq C_{l, R, H} k^{n-R},
$$

where the norm is computed in the space $C^{l}(X, \operatorname{End}(E))$ of End $(E)$-valued sections and $C_{l, R, H}$ depends on $l, R, \omega$ and $H$.

The first three coefficients $A_{0}, A_{1}$ and $A_{2}$ have been computed in [26, Theorem 4.2].

\section{Theorem 2.4 [26]}

(0) $A_{0}=I d$,

(1) $A_{1}=\sqrt{-1} \Lambda F_{H}+\frac{1}{2} \rho I d$,

(2)

$$
\begin{aligned}
A_{2}= & \frac{1}{3} \Delta \rho+\frac{1}{24}\left(|R|^{2}-4|R i c|^{2}+3 \rho^{2}\right) \\
& +\frac{1}{2}\left(\Delta^{\prime \prime} \operatorname{Ric}^{E}+\rho R i c^{E}+\operatorname{Ric}^{E} \operatorname{Ric}^{E}-R^{E} R^{E}-\left\langle R^{E}, R i c\right\rangle\right) .
\end{aligned}
$$

Here $R$, Ric and $\rho$ represent the curvature tensor, the Ricci curvature and the scalar curvature of $\omega$, and $\Delta=\phi^{\bar{j} i} \frac{\partial^{2}}{\partial v^{i} \partial \bar{v}^{j}}, R i c^{E}=\sqrt{-1} \Lambda F_{H}$ and $F_{H}$ represents the curvature of $(E, H)$, $\Delta^{\prime \prime}=\sqrt{-1} \Lambda \partial \bar{\partial}$.

When $E=K_{X}$ is the canonical bundle there is a natural metric $\left(\operatorname{det}\left(\phi_{i} \bar{j}\right)\right)^{-1}$ on $K_{X}$ induced from $\left(L, e^{-\phi}\right)$. In this case, $\operatorname{Ric}^{E}=-\rho, R^{E}=-$ Ric, so

$$
A_{0}=1, \quad A_{1}=-\frac{\rho}{2}, \quad A_{2}=-\frac{1}{6} \Delta^{\prime \prime} \rho+\frac{1}{24}\left(|R|^{2}-4|R i c|^{2}+3 \rho^{2}\right) .
$$

The Bergman kernel is

$$
B_{k}(H)=\sum_{j=1}^{d_{k}} u_{j}^{*} \otimes u_{j}=\sum_{j=1}^{d_{k}}\left|u_{j}\right|_{L^{2}}^{2}
$$


From (2.10), (2.11) and Theorem 2.3, the asymptotic expansion of Bergman kernel for the bundle $L^{k} \otimes K_{X}$ is

$$
\sum_{j=1}^{d_{k}}\left|u_{j}\right|_{L^{2}}^{2}=\frac{1}{(2 \pi)^{n}}\left(k^{n}-\frac{\rho}{2} k^{n-1}+\left(-\frac{1}{6} \Delta^{\prime \prime} \rho+\frac{1}{24}\left(|R|^{2}-4|R i c|^{2}+3 \rho^{2}\right)\right) k^{n-2}+\cdots\right) .
$$

\subsection{Analytic torsion and Quillen metric}

The definitions and results in this subsection can be found in [9,11-13,21,23].

Let $\pi: \mathcal{X} \rightarrow M$ be a proper holomorphic mapping between complex manifolds $\mathcal{X}$ and $M,\left(F, h_{F}\right)$ a holomorphic Hermitian vector bundle on $\mathcal{X}, \nabla^{F}$ the corresponding Chern connection, and $R^{F}=\left(\nabla^{F}\right)^{2}$ its curvature. For any $y \in M$, let $\mathcal{X}_{y}=\pi^{-1}(y)$ be the fiber over $y$ with Kähler metric $g^{\mathcal{X}_{y}}$ depending smoothly on $y$. The fibers are assumed to be compact.

For any $0 \leq p \leq n:=\operatorname{dim}_{\mathbb{C}} \mathcal{X}_{y}$ we put

$$
E_{y}^{p}:=A^{0, p}\left(\mathcal{X}_{y}, F\right), \quad E_{y}=\bigoplus_{p \geq 0} E_{y}^{p} .
$$

The operator $D_{y}=\bar{\partial}_{y}+\bar{\partial}_{y}^{*}$ acts on the fiber $E_{y}$.

For every $y \in M$, the spectrum of $D_{y}^{2}$ is discrete. For $b>0$, let $K_{y}^{b, p}$ be the sum of the eigenspaces of the operator $D_{y}^{2}$ acting on $E_{y}^{p}$ for eigenvalues $<b$. Let $U^{b}$ be the open set:

$$
U^{b}=\left\{y \in M ; b \notin \operatorname{Spec} D_{y}^{2}\right\} .
$$

On each open set $U^{b}, K^{b, p}$ is a smooth finite dimensional vector bundle. Set

$$
K^{b,+}=\bigoplus_{p \text { even }} K^{b, p}, \quad K^{b,-}=\bigoplus_{p \text { odd }} K^{b, p}, \quad K^{b}=K^{b,+} \oplus K^{b,-} .
$$

Define the following line bundle $\lambda^{b}$ on $U^{b}$,

$$
\lambda^{b}=\left(\operatorname{det} K^{b, 0}\right)^{-1} \otimes\left(\operatorname{det} K^{b, 1}\right) \otimes\left(\operatorname{det} K^{b, 2}\right)^{-1} \otimes \cdots .
$$

For $0<b<c$, if $y \in U^{b} \cap U^{c}$, let $K_{y}^{(b, c), p}$ be the sum of eigenspaces of $D_{y}^{2}$ in $E_{y}^{p}$ for eigenvalues $\mu$ such that $b<\mu<c$. Set

$$
K_{y}^{(b, c),+}=\bigoplus_{p \text { even }} K^{(b, c), p}, \quad K_{y}^{(b, c),-}=\bigoplus_{p \text { odd }} K^{(b, c), p}, \quad K_{y}^{(b, c)}=K_{y}^{(b, c),+} \oplus K_{y}^{(b, c),-} .
$$

Define $\lambda^{(b, c)}$ accordingly as before. Let $\bar{\partial}^{(b, c)}$ and $D^{(b, c)}$ be the restriction of $\bar{\partial}$ and $D$ to $K^{(b, c)} . D_{ \pm}^{(b, c)}$ is the restriction of $D$ to $K^{(b, c), \pm}$.

Since the chain complex

$$
0 \rightarrow K^{(b, c), 0} \stackrel{\bar{\partial}^{(b, c)}}{\longrightarrow} K^{(b, c), 1} \stackrel{\bar{\partial}^{(b, c)}}{\longrightarrow} \cdots \stackrel{\bar{\partial}^{(b, c)}}{\longrightarrow} K^{(b, c), n} \rightarrow 0
$$

is acyclic, $\lambda^{(b, c)}$ has a canonical non-zero section $T\left(\bar{\partial}^{(b, c)}\right)$ which is smooth on $U^{b} \cap U^{c}$ (see [11, Definition 1.1]). For $0<b<c$, over $U^{b} \cap U^{c}$, we have the $C^{\infty}$ identifications

$$
\lambda^{c}=\lambda^{b} \otimes \lambda^{(b, c)} \text {. }
$$


We identify $\lambda^{b}$ and $\lambda^{c}$ over $U^{b} \cap U^{c}$ by the $C^{\infty}$ map

$$
s \in \lambda^{b} \mapsto s \otimes T\left(\bar{\partial}^{(b, c)}\right) \in \lambda^{c} .
$$

Definition 2.5 [13, Def. 1.1] The $C^{\infty}$ line bundle $\lambda$ over $M$ is $\left\{\left(U^{b}, \lambda^{b}\right)\right\}$ with the transition functions (2.15) on $U^{b} \cap U^{c}$.

The analytic torsion of the chain complex (2.14) was introduced by Ray and Singer [23].

Definition 2.6 The analytic torsion $\tau\left(\bar{\partial}^{(b, c)}\right)$ associated to the acyclic chain complex (2.14) is defined as the positive real number

$$
\tau\left(\bar{\partial}^{(b, c)}\right)=\left(\left(\operatorname{det}\left(D_{1}^{(b, c)}\right)^{2}\right)\left(\operatorname{det}\left(D_{2}^{(b, c)}\right)^{2}\right)^{-2}\left(\operatorname{det}\left(D_{3}^{(b, c)}\right)^{2}\right)^{3} \cdots\right)^{1 / 2},
$$

where $D_{p}^{(b, c)}$ is the restriction of $D$ to $K^{(b, c), p}, 1 \leq p \leq n$. If $b$ is a small constant less than all positive eigenvalues of $D_{y}^{2}$, then we denote

$$
\tau(\bar{\partial}):=\tau\left(\bar{\partial}^{(b,+\infty)}\right) .
$$

Since $K^{b}$ and $K^{(b, c)}$ are orthogonal subspaces of $K^{c}$, by [11, Proposition 1.5], we find that if $s \in \lambda^{b}$,

$$
\left|s \otimes T\left(\bar{\partial}^{(b, c)}\right)\right|^{c}=|s|^{b} \tau\left(\bar{\partial}^{(b, c)}\right),
$$

where $|\cdot|^{b}$ is the induced metric by $\left(\mathcal{X}_{y}, g^{\mathcal{X}_{y}}\right)$ and $\left(F, h_{F}\right)$.

Now let $N_{V}$ be the number operator on $E$ such that $N_{V} \eta=p \eta$ for $\eta \in E^{p}$. Set $Q^{b}=$ $I-P^{b}$, where $P^{b}$ is the orthogonal projection operator from $E_{y}$ on $K_{y}^{b}$.

For $y \in U^{b}, \operatorname{Re}(s)>l$, set

$$
\theta_{y}^{b}(s)=-\operatorname{Tr}_{s}\left[N_{V}\left[D^{2}\right]^{-s} Q^{b}\right]=\frac{-1}{\Gamma(s)} \int_{0}^{+\infty} u^{s-1} \operatorname{Tr}_{s}\left[N_{V} \exp \left(-u D^{2}\right) Q^{b}\right] d u
$$

Similar if $0<b<c<+\infty$, for $y \in U^{b} \cap U^{c}$, set

$$
\theta_{y}^{(b, c)}(s)=-\operatorname{Tr}_{s}\left[N_{V}\left[D^{2}\right]^{-s} P^{(b, c)}\right]=\frac{-1}{\Gamma(s)} \int_{0}^{+\infty} u^{s-1} \operatorname{Tr}_{s}\left[N_{V} \exp \left(-u D^{2}\right) P^{(b, c)}\right] d u .
$$

The functions $\theta_{y}^{b}$ and $\theta_{y}^{(b, c)}$ extend into a meromorphic function which is holomorphic at $s=0$. Also on $U^{b} \cap U^{c}$,

$$
\theta^{b}=\theta^{(b, c)}+\theta^{c}
$$

and by [13, Equation 1.32],

$$
\log \left(\tau^{2}\left(\bar{\partial}^{(b, c)}\right)\right)=-\theta^{(b, c)^{\prime}}(0) .
$$

For $y \in U^{b}$, denote

$$
\tau_{y}\left(\bar{\partial}^{(b,+\infty)}\right)=\exp \left(-\frac{1}{2} \theta_{y}^{b^{\prime}}(0)\right) .
$$

Let $\|\bullet\|^{b}$ denote the metric on the line bundle $\left(\lambda^{b}, U^{b}\right)$,

$$
\|\bullet\|^{b}=|\bullet|^{b} \tau_{y}\left(\bar{\partial}^{(b,+\infty)}\right) .
$$

Then the definition of Quillen metric $\|\bullet\|_{Q}$ and Chern form $c_{1}\left(\lambda,\|\bullet\|_{Q}\right)$ of the Quillen metric are given by the following theorem. 
Theorem 2.7 [11-13] The metrics $\|\bullet\|^{b}$ on $\left(\lambda^{b}, U^{b}\right)$ patch into a smooth Hermitian metric $\|\bullet\|_{Q}$ on the holomorphic line bundle $\lambda$. The Chern form of Hermitian line bundle $\left(\lambda,\|\bullet\|_{Q}\right)$ is

$$
c_{1}\left(\lambda,\|\bullet\|_{Q}\right)=-\left\{\int_{\mathcal{X} / M} \operatorname{Td}\left(\frac{-R^{T_{\mathcal{X}} / M}}{2 \pi i}\right) \operatorname{Tr}\left[\exp \left(\frac{-R^{F}}{2 \pi i}\right)\right]\right\}^{(1,1)} .
$$

The Knudsen-Mumford determinant is defined by

$$
\lambda^{K M}=\left(\operatorname{det} R \pi_{*} F\right)^{-1} .
$$

The fiber $\lambda_{y}^{K M}$ is by definition given by

$$
\lambda_{y}^{K M}=\bigotimes_{i \geq 0} \operatorname{det} H^{i}\left(\mathcal{X}_{y}, F\right)^{(-1)^{i+1}}
$$

We assume that $\pi$ is locally Kähler, i.e. there is an open covering $\mathscr{U}$ of $M$ such that if $U \in \mathscr{U}, \pi^{-1}(U)$ admits a Kähler metric.

Theorem 2.8 [11-13] Assume that $\pi$ is locally Kähler. Then the identification of the fibers $\lambda_{y} \cong \lambda_{y}^{K M}$ defines a holomorphic isomorphism of line bundles $\lambda \cong \lambda^{K M}$. The Chern form of the Quillen metric on $\lambda \cong \lambda^{K M}$ is given by (2.17).

\section{The asymptotic of the curvature of direct image bundle}

We shall give the expansion of $c_{1}\left(E^{k},\|\bullet\|_{k}\right)$ and $-c_{1}\left(\lambda,\|\bullet\|_{Q}\right)$ up to $o\left(k^{n-1}\right)$.

Let $\pi: \mathcal{X} \rightarrow M$ be a holomorphic fibration with compact fibers and $L$ a relatively ample line bundle over $\mathcal{X}$ as in the Sect. 2.1. Denote

$$
\omega=\sqrt{-1} \partial \bar{\partial} \phi
$$

\subsection{The asymptotic of the curvature of $L^{2}$-metric}

The curvature of direct image bundle $E^{k}=\pi_{*}\left(L^{k} \otimes K_{\mathcal{X} / M}\right)$ is, by (2.7),

$$
\left\langle\sqrt{-1} \Theta^{E^{k}} u, u\right\rangle=\int_{\mathcal{X}_{y}} k c(\phi)|u|^{2} e^{-k \phi}+k\left\langle\left(k+\Delta^{\prime}\right)^{-1} i_{\mu_{\alpha}} u, i_{\mu_{\beta}} u\right\rangle \sqrt{-1} d z^{\alpha} \wedge d \bar{z}^{\beta}
$$

for any element $u \in E_{y}^{k}$. For any vector $\zeta=\zeta^{\alpha} \frac{\partial}{\partial z^{\alpha}}$ of $T M$,

$$
\begin{aligned}
\left\langle\Theta^{E^{k}} u, u\right\rangle(\zeta, \bar{\zeta})= & -\sqrt{-1}\left(\int_{\mathcal{X}_{y}} k c(\phi)|u|^{2} e^{-k \phi}\right)(\zeta, \bar{\zeta}) \\
& +k\left\langle\left(k+\Delta^{\prime}\right)^{-1} i_{\mu} u, i_{\mu} u\right\rangle
\end{aligned}
$$

where

$$
\mu=\mu_{\bar{j}}^{i} d \bar{v}^{j} \otimes \frac{\partial}{\partial v^{i}}, \quad \mu_{\bar{j}}^{i}=-\partial_{\bar{j}}\left(\phi_{\alpha \bar{l}} \phi^{\bar{l} i}\right) \zeta^{\alpha} .
$$

The following technical expansion of $\left(\Delta^{\prime}+k\right)^{-1}$ will be critical to find the asymptotics of the $L^{2}$-curvature; the main point of this expansion is that the leading term of the contribution 
to the $L^{2}$-curvature of each term below is effectively found. (Composed with the operators $i_{\mu}$ and $i_{\mu}^{*}$ it gives an expansion of the Toeplitz operator with symbol $i_{\mu}^{*}\left(\Delta^{\prime}+k\right)^{-1} i_{\mu}$ on the cohomology space $H^{0}\left(\mathcal{X}_{y}, L^{k} \otimes K_{\mathcal{X}_{y}}\right)$.)

Lemma 3.1 The resolvent operator $\left(\Delta^{\prime}+k\right)^{-1}$ has the following 7-term-expansion,

$$
\left(\Delta^{\prime}+k\right)^{-1}=I+I I+\cdots+V I+V I I,
$$

where

$$
\begin{aligned}
I & =\frac{1}{2 k}, \quad I I=\frac{1}{6 k^{2}}\left(k-\Delta^{\prime}-R^{*}\right), \quad I I I=\frac{1}{4 k^{2}} R^{*}, I V=\frac{1}{4 k^{2}}\left(\Delta^{\prime}+k\right)^{-1}\left(k-\Delta^{\prime}\right) R^{*}, \\
V & =\frac{1}{18 k^{3}}\left(2 k-\Delta^{\prime}\right)\left(k-\Delta^{\prime}-R^{*}\right), V I=\frac{1}{36 k^{4}}\left(2 k-\Delta^{\prime}\right)^{2}\left(k-\Delta^{\prime}-R^{*}\right), \\
V I I & =\frac{1}{36 k^{4}}\left(\Delta^{\prime}+k\right)^{-1}\left(k-\Delta^{\prime}\right)\left(2 k-\Delta^{\prime}\right)^{2}\left(k-\Delta^{\prime}-R^{*}\right) .
\end{aligned}
$$

Proof The RHS of (3.4), by elementary computations, is

$$
\begin{aligned}
\frac{1}{2 k}+ & \frac{1}{6 k^{2}}\left(k-\Delta^{\prime}-R^{*}\right)+\frac{1}{4 k^{2}} R^{*}+\frac{1}{4 k^{2}}\left(\Delta^{\prime}+k\right)^{-1}\left(k-\Delta^{\prime}\right) R^{*} \\
& +\frac{1}{18 k^{3}}\left(2 k-\Delta^{\prime}\right)\left(k-\Delta^{\prime}-R^{*}\right)+\frac{1}{36 k^{4}}\left(2 k-\Delta^{\prime}\right)^{2}\left(k-\Delta^{\prime}-R^{*}\right) \\
& +\frac{1}{36 k^{4}}\left(\Delta^{\prime}+k\right)^{-1}\left(k-\Delta^{\prime}\right)\left(2 k-\Delta^{\prime}\right)^{2}\left(k-\Delta^{\prime}-R^{*}\right) \\
= & \frac{1}{2 k}+\frac{1}{6 k^{2}}\left(k-\Delta^{\prime}-R^{*}\right)+\frac{1}{4 k^{2}}\left(\left(\Delta^{\prime}+k\right)^{-1}\left(k-\Delta^{\prime}\right)+I d\right) R^{*} \\
& +\frac{1}{18 k^{3}}\left(2 k-\Delta^{\prime}\right)\left(k-\Delta^{\prime}-R^{*}\right) \\
& +\frac{1}{36 k^{4}}\left(\left(\Delta^{\prime}+k\right)^{-1}\left(k-\Delta^{\prime}\right)+I d\right)\left(2 k-\Delta^{\prime}\right)^{2}\left(k-\Delta^{\prime}-R^{*}\right) \\
= & \frac{1}{2 k}+\frac{1}{6 k^{2}}\left(k-\Delta^{\prime}-R^{*}\right)+\frac{1}{2 k}\left(\Delta^{\prime}+k\right)^{-1} R^{*} \\
& +\frac{1}{18 k^{3}}\left(2 k-\Delta^{\prime}\right)\left(k-\Delta^{\prime}-R^{*}\right) \\
& +\frac{1}{18 k^{3}}\left(\Delta^{\prime}+k\right)^{-1}\left(2 k-\Delta^{\prime}\right)^{2}\left(k-\Delta^{\prime}-R^{*}\right) \\
= & \frac{1}{2 k}+\frac{1}{6 k^{2}}\left(k-\Delta^{\prime}-R^{*}\right)+\frac{1}{2 k}\left(\Delta^{\prime}+k\right)^{-1} R^{*} \\
& +\frac{1}{6 k^{2}}\left(\Delta^{\prime}+k\right)^{-1}\left(2 k-\Delta^{\prime}\right)\left(k-\Delta^{\prime}-R^{*}\right) \\
= & \frac{1}{2 k}+\frac{1}{2 k}\left(\Delta^{\prime}+k\right)^{-1}\left(k-\Delta^{\prime}-R^{*}\right)+\frac{1}{2 k}\left(\Delta^{\prime}+k\right)^{-1} R^{*} \\
= & \frac{1}{2 k}+\frac{1}{2 k}\left(\Delta^{\prime}+k\right)^{-1}\left(k-\Delta^{\prime}\right) \\
= & \left(\Delta^{\prime}+k\right)^{-1},
\end{aligned}
$$

which completes the proof. Here the second and last equalities follow from

$$
\left(\Delta^{\prime}+k\right)^{-1}\left(k-\Delta^{\prime}\right)+I d=2 k\left(\Delta^{\prime}+k\right)^{-1} .
$$


while the third and fourth equalities follow from

$$
\left(\Delta^{\prime}+k\right)^{-1}\left(2 k-\Delta^{\prime}\right)+I d=3 k\left(\Delta^{\prime}+k\right)^{-1} .
$$

We shall treat each term in the expansion using the following lemmas. We refer [15, Chapter VII] for the calculus on Kähler manifolds.

Lemma 3.2 Let $(X, \omega)$ be a compact Kähler manifold and $\left(L^{k}, e^{-k \phi}\right)$ be a Hermitian line bundle over $X$ with $\sqrt{-1} \partial \bar{\partial} \phi=\omega$. For any $\alpha \in A^{n-1,1}\left(X, L^{k}\right)$ it holds

$$
\left(k-\nabla^{*} \nabla^{\prime}-R^{*}\right) \alpha=\left(d v^{t}\right)^{*} \nabla^{\prime}\left(\nabla_{\frac{\partial}{\partial \bar{v}^{t}}} \alpha\right),
$$

where $\nabla^{*}$ is the adjoint operator of the $(1,0)$-part $\nabla^{\prime}$ of Chern connection, $\left(d v^{t}\right)^{*}=\phi^{\bar{t} s} i \frac{\partial}{\partial v^{s}}$, $R^{*}=R_{i \bar{l}}^{\bar{t} j} i_{\frac{\partial}{\partial v^{j}}} d v^{i} \wedge d \bar{v}^{l} \wedge i_{\frac{\partial}{\partial \bar{v}^{t}}}, R_{i \bar{l}}^{\bar{t} j}=-\partial_{i} \partial_{\bar{l}} \phi^{\bar{t} j}+\phi_{\bar{l}}^{\bar{t} s} \phi_{i}^{\bar{k} j} \phi_{s \bar{k}}$ is the Chern curvature component of $\left(T_{X}^{*},\left(\phi^{i} \bar{j}\right)\right)$, and $\nabla_{\frac{\partial}{\partial \bar{v}^{t}}}=\frac{\partial}{\partial \bar{v}^{t}}-\bar{\Gamma}_{t j}^{l} d \bar{v}^{j} \wedge i_{\frac{\partial}{\partial \bar{v}^{l}}}, \Gamma_{t j}^{l}=\frac{\partial \phi_{t \bar{k}}}{\partial v^{j}} \phi^{k l}$.

Proof By [15, Chapter VII, Theorem (1.1)], $\nabla^{*}=\sqrt{-1}[\Lambda, \bar{\partial}]$, where $\Lambda$ is the adjoint of multiplication operator $\omega \wedge \bullet$ by the Kähler form. Thus

$$
\begin{aligned}
\nabla^{\prime *} \nabla^{\prime} \alpha & =\sqrt{-1}[\Lambda, \bar{\partial}] \nabla^{\prime} \alpha \\
& =\sqrt{-1} \Lambda \bar{\partial} \nabla^{\prime} \alpha-\sqrt{-1} \bar{\partial} \Lambda \nabla^{\prime} \alpha .
\end{aligned}
$$

We expand the second term and find

$$
\begin{aligned}
& \sqrt{-1} \bar{\partial} \Lambda \nabla^{\prime} \alpha=\bar{\partial}\left(\phi^{s \bar{t}} i_{\frac{\partial}{\partial \bar{v}^{t}}} i_{\frac{\partial}{\partial v^{s}}} \nabla^{\prime} \alpha\right) \\
& =\left(\bar{\partial} \phi^{s \bar{t}}\right) \wedge i_{\frac{\partial}{\partial \bar{v}^{t}}} i_{\frac{\partial}{\partial v^{s}}} \nabla^{\prime} \alpha+\phi^{s \bar{t}} i_{\frac{\partial}{\partial \bar{v}^{t}}} i_{\frac{\partial}{\partial v^{s}}} \bar{\partial} \nabla^{\prime} \alpha+\phi^{s \bar{t}} \frac{\partial}{\partial \bar{v}^{t}} i_{\frac{\partial}{\partial v^{s}}} \nabla^{\prime} \alpha \\
& =\left(\bar{\partial} \phi^{s \bar{t}}\right) \wedge i_{\frac{\partial}{\partial v^{s}}} \nabla^{\prime} i_{\frac{\partial}{\partial \bar{v}^{t}}} \alpha+\sqrt{-1} \Lambda \bar{\partial} \nabla^{\prime} \alpha+\phi^{s \bar{t}} i_{\frac{\partial}{\partial v^{s}}} \nabla^{\prime} \frac{\partial \alpha}{\partial \bar{v}^{t}}-k \alpha,
\end{aligned}
$$

where the first equality holds since $\left[\bar{\partial}, i_{\frac{\partial}{\partial \bar{v}^{t}}}\right]=\frac{\partial}{\partial \bar{v}^{t}}$ and $\left[\bar{\partial}, i_{\frac{\partial}{\partial v^{i}}}\right]=0$, the second equality follows from $\left[\frac{\partial}{\partial v^{t}}, \nabla^{\prime}\right]=-k \partial \phi_{\bar{t}}$ and $\left[i \frac{\partial}{\partial \bar{v}^{t}}, i_{\frac{\partial}{\partial v^{s}}}\right]=\left[i_{\frac{\partial}{\partial \bar{v}^{t}}}, \nabla^{\prime}\right]=0$. Combining (3.5) with (3.6) we obtain

$$
\left(k-\nabla^{*} \nabla\right) \alpha=\left(\bar{\partial} \phi^{s \bar{t}}\right) \wedge i_{\frac{\partial}{\partial v^{s}}} \nabla^{\prime} i_{\frac{\partial}{\partial \bar{v}^{t}}} \alpha+\phi^{s \bar{t}} i_{\frac{\partial}{\partial v^{s}}} \nabla^{\prime} \frac{\partial \alpha}{\partial \bar{v}^{t}} .
$$

Furthermore the action on $\alpha$ of the second term in the RHS of (3.7), by the definition of $\nabla \frac{\partial}{\partial \bar{v}^{t}}$, is the operator

$$
\begin{aligned}
& \phi^{s \bar{t}} i_{\frac{\partial}{\partial v^{s}}} \nabla^{\prime} \frac{\partial}{\partial \bar{v}^{t}} \\
& =\phi^{s \bar{t}} i_{\frac{\partial}{\partial v^{s}}} \nabla^{\prime} \nabla_{\frac{\partial}{\partial \bar{v}^{t}}}+\phi^{s \bar{t}} i_{\frac{\partial}{\partial v^{s}}} \nabla^{\prime}\left(\overline{\Gamma_{t l}^{k}} d \bar{v}^{l} \wedge i_{\frac{\partial}{\partial \bar{v}^{k}}}\right) \\
& =\left(d v^{t}\right)^{*} \nabla^{\prime} \nabla_{\frac{\partial}{\partial \bar{v}^{t}}}+\phi^{s \bar{t}}\left(\partial_{i} \overline{\Gamma_{t l}^{k}}\right) i_{\frac{\partial}{\partial v^{s}}} d v^{i} \wedge d \bar{v}^{l} \wedge i_{\frac{\partial}{\partial \bar{v}^{k}}}+\phi^{s \bar{t}} \overline{\Gamma_{t l}^{k}} d \bar{v}^{l} \wedge i_{\frac{\partial}{\partial v^{s}}} \nabla^{\prime} i_{\frac{\partial}{\partial \bar{v}^{k}}} \\
& =\left(d v^{t}\right)^{*} \nabla^{\prime} \nabla_{\frac{\partial}{\partial \bar{v}^{t}}}+R^{*}-\left(\bar{\partial} \phi^{s \bar{t}}\right) \wedge i_{\frac{\partial}{\partial v^{s}}} \nabla^{\prime} i_{\frac{\partial}{\partial \bar{v}^{t}}} .
\end{aligned}
$$


Substituting (3.8) into (3.7) we have

$$
\left(k-\nabla^{*} \nabla^{\prime}-R^{*}\right) \alpha=\left(d v^{t}\right)^{*} \nabla^{\prime}\left(\nabla_{\frac{\partial}{\partial \bar{v}^{t}}} \alpha\right) .
$$

Lemma 3.3 The following identity holds,

$$
\left\langle\left(k-\Delta^{\prime}\right) i_{\mu} u, i_{\mu} u\right\rangle=\int_{\mathcal{X}_{y}}\left(|\mu|_{R^{*}}^{2}-|\bar{\nabla} \mu|^{2}\right)|u|^{2} e^{-k \phi},
$$

where we have introduced $|\mu|_{R^{*}}^{2}:=\mu_{\bar{t}}^{l} R_{i \bar{l}}^{\bar{i} j} \overline{\mu_{\bar{j}}^{i}}$, which need not to be nonnegative, $|\bar{\nabla} \mu|^{2}=$ $\nabla_{\bar{l}} \mu_{\bar{t}}^{i} \overline{\nabla_{\bar{k}} \mu_{\bar{q}}^{s}} \phi_{i \bar{s}} \phi^{\bar{t} q} \phi^{\bar{l} k}, \nabla_{\bar{l}} \mu_{\bar{t}}^{i}=\partial_{\bar{l}} \mu_{\bar{t}}^{i}-\overline{\Gamma_{t l}^{s}} \mu_{\bar{s}}^{i}$.

Proof By a direct computation, we find

$$
\begin{aligned}
i_{\mu} u & =\left(-\partial_{\bar{l}}\left(\phi^{i} \bar{j} \phi_{\alpha \bar{j}}\right) \zeta^{\alpha}\right) d \bar{v}^{l} \wedge i_{\frac{\partial}{\partial v^{i}}}\left(u^{\prime} d v \otimes e^{k}\right) \\
& =\partial_{\bar{l}}\left(\phi^{i} \bar{j} \phi_{\alpha \bar{j}}\right) \zeta^{\alpha} u^{\prime} d \bar{v}^{l} \wedge(-1)^{i} d v^{1} \wedge \cdots \widehat{d v^{i}} \cdots \wedge d v^{n} \otimes e^{k} \\
& =\nabla^{\prime *}\left(\phi_{\alpha \bar{j}} \zeta^{\alpha} u^{\prime} d \bar{v}^{j} \wedge d v \otimes e^{k}\right) \\
& =\nabla^{\prime *}\left(\phi_{\alpha \bar{j}} \zeta^{\alpha} d \bar{v}^{j} \wedge u\right) .
\end{aligned}
$$

It follows that

$$
\Delta^{\prime} i_{\mu} u=\left(\nabla^{* *} \nabla^{\prime}+\nabla^{\prime} \nabla^{* *}\right) i_{\mu} u=\nabla^{*} \nabla^{\prime} i_{\mu} u .
$$

Thus, using Lemma 3.2 and (3.9), we obtain

$$
\begin{aligned}
\left\langle\left(k-\Delta^{\prime}\right) i_{\mu} u, i_{\mu} u\right\rangle & =\left\langle\left(k-\nabla^{*} \nabla\right) i_{\mu} u, i_{\mu} u\right\rangle \\
& =\left\langle\left(d v^{t}\right)^{*} \nabla^{\prime}\left(\nabla \frac{\partial}{\partial \bar{v}^{t}} i_{\mu} u\right)+R^{*} i_{\mu} u, i_{\mu} u\right\rangle \\
& =\left\langle R^{*} i_{\mu} u, i_{\mu} u\right\rangle+\left\langle\nabla_{\frac{\partial}{\partial \bar{v}^{t}}} i_{\mu} u, \nabla^{*}\left(d v^{t} \wedge i_{\mu} u\right)\right\rangle .
\end{aligned}
$$

In terms of local coordinates the first term is

$$
\begin{aligned}
\left\langle R^{*} i_{\mu} u, i_{\mu} u\right\rangle & =\left\langle R_{i \bar{l}}^{\bar{t} j} i_{\frac{\partial}{\partial v^{j}}} d v^{i} \wedge d \bar{v}^{l} \wedge i_{\frac{\partial}{\partial \bar{v}^{t}}} \mu_{\bar{q}}^{s} d \bar{v}^{q} \wedge i_{\frac{\partial}{\partial v^{s}}} u, i_{\mu} u\right\rangle \\
& =\left\langle R_{i \bar{l}}^{\bar{t} j} \mu_{\bar{t}}^{i} d \bar{v}^{l} \wedge i_{\frac{\partial}{\partial v^{j}}} u, \mu_{\bar{k}}^{s} d \bar{v}^{k} \wedge i_{\frac{\partial}{\partial v^{s}}} u\right\rangle \\
& =\int_{\mathcal{X}_{y}}\left(R_{i \bar{l}}^{\bar{t} j} \mu_{\bar{t}}^{i} \overline{\mu_{\bar{k}}^{s}} \phi^{\bar{l} k} \phi_{j \bar{s}}\right)|u|^{2} e^{-k \phi} \\
& =\int_{\mathcal{X}_{y}}\left(R_{i \bar{l}}^{\bar{t} j} \mu_{\bar{t}}^{i} \mu_{\bar{j}}^{\bar{l}}\right)|u|^{2} e^{-k \phi}=\int_{\mathcal{X}_{y}}|\mu|_{R^{*}}^{2}|u|^{2} e^{-k \phi}
\end{aligned}
$$


where the fourth equality follows from the definition of $\mu$ (3.3) and

$$
\begin{aligned}
\overline{\mu_{\bar{k}}^{s}} \phi^{\bar{l} k} \phi_{j \bar{s}} & =-\overline{\zeta^{\alpha}} \partial_{k}\left(\phi_{\bar{\alpha} i} \phi^{i \bar{s}}\right) \phi^{\bar{l} k} \phi_{j \bar{s}} \\
& =-\overline{\zeta^{\alpha}}\left(\phi_{\bar{\alpha} i k} \phi^{i \bar{s}}+\phi_{\bar{\alpha} i} \phi_{k}^{i \bar{s}}\right) \phi^{\bar{l} k} \phi_{j \bar{s}} \\
& =-\overline{\zeta^{\alpha}} \phi_{\bar{\alpha} i k} \phi^{i \bar{s}} \phi^{\bar{l} k} \phi_{j \bar{s}}+\overline{\zeta^{\alpha}} \phi_{\bar{\alpha} i} \phi^{i \bar{s}} \phi_{k j \bar{s}} \phi^{\bar{l} k} \\
& =-\overline{\zeta^{\alpha}} \phi_{\bar{\alpha} j k} \phi^{\bar{l} k}-\overline{\zeta^{\alpha}} \phi_{\bar{\alpha} i} \phi_{j}^{i \bar{s}} \phi_{k \bar{s}} \phi^{\bar{l} k} \\
& =-\overline{\zeta^{\alpha}} \phi_{\bar{\alpha} j i} \phi^{\overline{l i}}-\overline{\zeta^{\alpha}} \phi_{\bar{\alpha} i} \phi_{j}^{i \bar{l}} \\
& =-\overline{\zeta^{\alpha}} \partial_{j}\left(\phi_{\bar{\alpha} i} \phi^{\bar{l} i}\right)=\overline{\mu_{\bar{j}}^{l}} .
\end{aligned}
$$

The second term in the RHS of (3.10) is

$$
\begin{aligned}
\left\langle\nabla_{\frac{\partial}{\partial \bar{v}^{t}}} i_{\mu} u, \nabla^{*}\left(d v^{t} \wedge i_{\mu} u\right)\right\rangle \\
=\left\langle\left(\nabla_{\bar{t}} \mu_{\bar{j}}^{i}\right) d \bar{v}^{j} \wedge i_{\frac{\partial}{\partial v^{i}}} u,-\bar{\partial}\left(\phi^{s \bar{l}} \mu_{\bar{l}}^{t}\right) i_{\frac{\partial}{\partial v^{s}}} u\right\rangle \\
=-\left\langle\left(\bar{\partial}\left(\phi^{s \bar{l}} \mu_{\bar{l}}^{t}\right) i_{\frac{\partial}{\partial v^{s}}}\right)^{*}\left(\nabla_{\bar{t}} \mu_{\bar{j}}^{i}\right) d \bar{v}^{j} \wedge i_{\frac{\partial}{\partial v^{i}}} u, u\right\rangle \\
=-\left\langle\phi_{i \bar{s}} \partial_{k}\left(\phi^{\bar{s} l} \overline{\mu_{\bar{l}}^{t}}\right) \phi^{\bar{j} k}\left(\nabla_{\bar{t}} \mu_{\bar{j}}^{i}\right) u, u\right\rangle \\
=\int_{\mathcal{X}_{y}}-\nabla_{k} \overline{\mu_{\bar{i}}^{t}} \phi^{\bar{j} k} \nabla_{\bar{t}} \mu_{\bar{j}}^{i}|u|^{2} e^{-k \phi} \\
=\int_{\mathcal{X}_{y}}-\nabla_{k} \overline{\mu_{\bar{i}}^{t}} \phi^{\bar{j} k} \nabla_{\bar{j}} \mu_{\bar{t}}^{i}|u|^{2} e^{-k \phi} \\
=\int_{\mathcal{X}_{y}}-\nabla_{k} \overline{\mu_{\bar{i}}^{t}} \phi^{\bar{j} k} \nabla_{\bar{j}} \mu_{\bar{q}}^{s} \phi^{\bar{q} i} \phi_{s \bar{t}}|u|^{2} e^{-k \phi}=\int_{\mathcal{X}_{y}}-|\bar{\nabla} \mu|^{2}|u|^{2} e^{-k \phi},
\end{aligned}
$$

where in the second equality, $(\bullet)^{*}$ denotes the adjoint operator of $\bullet$, the fifth equality follows from

$$
\nabla_{\bar{t}} \mu_{\bar{j}}^{i}=\nabla_{\bar{t}}\left(\partial_{\bar{j}}\left(\phi_{\alpha \bar{l}} \phi^{\overline{l i}}\right) \zeta^{\alpha}\right)=\partial_{\bar{t}} \partial_{\bar{j}}\left(\phi_{\alpha \bar{l}} \phi^{\overline{l i}}\right) \zeta^{\alpha}-\overline{\Gamma_{t j}^{s}} \mu_{\bar{s}}^{i}=\nabla_{\bar{j}} \mu_{\bar{t}}^{i}
$$

The sixth equality holds by (3.12) and $\nabla_{\bar{j}}\left(\phi_{s \bar{t}}\right)=\nabla_{\bar{j}}\left(\phi^{\bar{q} i}\right)=0$. Substituting (3.11) and (3.13) into (3.10) we have

$$
\left\langle\left(k-\Delta^{\prime}\right) i_{\mu} u, i_{\mu} u\right\rangle=\int_{\mathcal{X}_{y}}\left(|\mu|_{R^{*}}^{2}-|\bar{\nabla} \mu|^{2}\right)|u|^{2} e^{-k \phi} .
$$

In the subsequent text we shall write $O\left(k^{j}\right)$ for any term that is of the order $k^{j}$ and is independent of $u$.

Lemma 3.4 We have the following expansion

$$
\left\langle\left(k-\Delta^{\prime}\right)^{2} i_{\mu} u, i_{\mu} u\right\rangle=\int_{\mathcal{X}_{y}}\left(k|\bar{\nabla} \mu|^{2}+O(1)\right)|u|^{2} e^{-k \phi} .
$$


Proof Writing $k-\Delta^{\prime}=\left(k-\Delta^{\prime}-R^{*}\right)+R^{*}$ and using Lemma 3.2 we have

$$
\begin{aligned}
\left\langle\left(k-\Delta^{\prime}\right)^{2} i_{\mu} u, i_{\mu} u\right\rangle=\left\|\left(k-\Delta^{\prime}\right) i_{\mu} u\right\|^{2} \\
=\left\|\phi^{s \bar{t}} i_{\frac{\partial}{\partial v^{s}}} \nabla^{\prime}\left(\nabla_{\frac{\partial}{\partial \bar{t}^{t}}} i_{\mu} u\right)+R^{*} \alpha\right\|^{2} \\
=\left\|\phi^{s \bar{t}} i_{\frac{\partial}{\partial v^{s}}} \nabla^{\prime}\left(\nabla_{\frac{\partial}{\partial \bar{v}^{t}}} i_{\mu} u\right)\right\|^{2}+\left\|R^{*} i_{\mu} u\right\|^{2}+2 \operatorname{Re}\left\langle\phi^{s \bar{t}} i_{\frac{\partial}{\partial v^{s}}} \nabla^{\prime}\left(\nabla_{\frac{\partial}{\partial \bar{v}^{t}}} i_{\mu} u\right), R^{*} i_{\mu} u\right\rangle \\
=\left\langle\nabla^{\prime}\left(\nabla_{\frac{\partial}{\partial \bar{v}^{j}}} i_{\mu} u\right), d v^{j} \wedge \phi^{s \bar{t}} i_{\frac{\partial}{\partial v^{s}}} \nabla^{\prime}\left(\nabla_{\frac{\partial}{\partial \bar{v}^{t}}} i_{\mu} u\right)\right\rangle+\left\|R^{*} i_{\mu} u\right\|^{2} \\
\quad+2 \operatorname{Re}\left\langle\nabla^{\prime}\left(\nabla_{\frac{\partial}{\partial \bar{v}^{t}}} i_{\mu} u\right), d v^{t} \wedge R^{*} i_{\mu} u\right\rangle \\
=\left\langle\nabla_{\frac{\partial}{\partial \bar{v}^{j}}} i_{\mu} u, \nabla^{*}\left(\phi^{j \bar{t}} \nabla^{\prime}\left(\nabla_{\frac{\partial}{\partial \bar{v}^{t}}} i_{\mu} u\right)\right)\right\rangle+\left\|R^{*} i_{\mu} u\right\|^{2}+2 \operatorname{Re}\left\langle\nabla_{\frac{\partial}{\partial \bar{v}^{t}}} i_{\mu} u, \nabla^{*}\left(d v^{t} \wedge R^{*} i_{\mu} u\right)\right\rangle .
\end{aligned}
$$

For the first term in the RHS of (3.14), we have

$$
\begin{aligned}
& \left\langle\nabla_{\frac{\partial}{\partial \bar{v}^{j}}} i_{\mu} u, \nabla^{*}\left(\phi^{j \bar{t}} \nabla^{\prime}\left(\nabla_{\frac{\partial}{\partial \bar{v}^{t}}} i_{\mu} u\right)\right)\right\rangle \\
& =\left\langle\nabla_{\frac{\partial}{\partial \bar{v}^{j}}} i_{\mu} u,\left(\phi^{j \bar{t}} \nabla^{\prime *}-\phi^{s \bar{l}} \phi_{\bar{l}}^{j \bar{t}} i_{\frac{\partial}{\partial v^{s}}}\right) \nabla^{\prime}\left(\nabla_{\frac{\partial}{\partial \bar{v}^{t}}} i_{\mu} u\right)\right\rangle \\
& =-\left\langle\nabla^{\prime *}\left(\phi_{l}^{\bar{j} t} d v^{l} \wedge \nabla_{\frac{\partial}{\partial \bar{v}^{j}}} i_{\mu} u\right), \nabla_{\frac{\partial}{\partial \bar{v}^{t}}} i_{\mu} u\right\rangle+\left\langle\nabla_{\frac{\partial}{\partial \bar{v}^{j}}} i_{\mu} u, \phi^{j \bar{t}} \nabla^{\prime *} \nabla^{\prime}\left(\nabla_{\frac{\partial}{\partial \bar{v}^{t}}} i_{\mu} u\right)\right\rangle .
\end{aligned}
$$

For the second term in RHS of (3.15), using Lemma 3.2, we obtain

$$
\begin{aligned}
&\left\langle\nabla_{\frac{\partial}{\partial \bar{v}^{j}}} i_{\mu} u, \phi^{j \bar{t}} \nabla^{\prime *} \nabla^{\prime}\left(\nabla_{\frac{\partial}{\partial \bar{v}^{t}}} i_{\mu} u\right)\right\rangle \\
&=\left\langle\nabla_{\frac{\partial}{\partial \bar{v}^{j}}} i_{\mu} u, \phi^{j \bar{t}}\left(k-\phi^{s \bar{l}} i_{\frac{\partial}{\partial v^{s}}} \nabla^{\prime} \nabla_{\frac{\partial}{\partial \bar{v}^{l}}}-R^{*}\right)\left(\nabla_{\frac{\partial}{\partial \bar{v}^{t}}} i_{\mu} u\right)\right\rangle \\
&=\int_{\mathcal{X}_{y}} k \phi^{\bar{j} t} \phi^{\bar{l} s} \phi_{k \bar{i}} \nabla_{\bar{j}} \mu_{\bar{l}}^{k} \nabla_{t} \overline{\mu_{\bar{s}}^{i}}|u|^{2} e^{-\phi}-\left\langle\nabla^{*}\left(d v^{l} \wedge \nabla_{\frac{\partial}{\partial \bar{v}^{j}}} i_{\mu} u\right), \nabla_{\frac{\partial}{\partial \bar{v}^{j}}} \nabla_{\frac{\partial}{\partial \bar{v}^{t}}} i_{\mu} u\right\rangle \\
&-\left\langle\nabla_{\frac{\partial}{\partial \bar{v}^{j}}} i_{\mu} u, \phi^{j \bar{t}} R^{*}\left(\nabla_{\frac{\partial}{\partial \bar{v}^{t}}} i_{\mu} u\right)\right\rangle .
\end{aligned}
$$

We substitute (3.15) and (3.16) into (3.14),

$$
\begin{aligned}
\langle(k & \left.\left.-\Delta^{\prime}\right)^{2} i_{\mu} u, i_{\mu} u\right\rangle=\left\|R^{*} i_{\mu} u\right\|^{2}+2 \operatorname{Re}\left\langle\nabla_{\frac{\partial}{\partial \bar{v}^{t}}} i_{\mu} u, \nabla^{*}\left(d v^{t} \wedge R^{*} i_{\mu} u\right)\right\rangle \\
& -\left\langle\nabla^{*}\left(\phi_{l}^{\bar{j} t} d v^{l} \wedge \nabla_{\frac{\partial}{\partial \bar{v}^{j}}} i_{\mu} u\right), \nabla_{\frac{\partial}{\partial \bar{v}^{t}}} i_{\mu} u\right\rangle-\left\langle\nabla^{*}\left(d v^{l} \wedge \nabla_{\frac{\partial}{\partial \bar{v}^{j}}} i_{\mu} u\right), \nabla_{\frac{\partial}{\partial \bar{v}^{j}}} \nabla_{\frac{\partial}{\partial \bar{v}^{t}}} i_{\mu} u\right\rangle \\
& -\left\langle\nabla_{\frac{\partial}{\partial \bar{v}^{j}}} i_{\mu} u, \phi^{j \bar{t}} R^{*}\left(\nabla_{\frac{\partial}{\partial \bar{v}^{t}}} i_{\mu} u\right)\right\rangle+\int_{\mathcal{X}_{y}} k \phi^{\bar{j} t} \phi^{\bar{l} s} \phi_{k \bar{i}} \nabla_{\bar{j}} \mu_{\bar{l}}^{k} \nabla_{t} \overline{\mu_{\bar{s}}^{i}}|u|^{2} e^{-k \phi} \\
= & \int_{\mathcal{X}_{y}}\left(k|\bar{\nabla} \mu|^{2}+O(1)\right)|u|^{2} e^{-k \phi} .
\end{aligned}
$$

This completes the proof. 
Lemma 3.5 The quadratic form $\left\langle\left(k-\Delta^{\prime}\right)^{3} i_{\mu} u, i_{\mu} u\right\rangle$ has the following expansion

$$
\left\langle\left(k-\Delta^{\prime}\right)^{3} i_{\mu} u, i_{\mu} u\right\rangle=\int_{\mathcal{X}_{y}}\left(-k^{2}|\bar{\nabla} u|^{2}+O(k)\right)|u|^{2} e^{-k \phi} .
$$

Proof Denote $\nabla_{\bar{t}}=\nabla_{\frac{\partial}{\partial \bar{v}^{t}}}$. We have, using Lemma 3.2, that

$$
\begin{aligned}
\left\langle\left(k-\Delta^{\prime}\right)^{3} i_{\mu} u, i_{\mu} u\right\rangle & \\
= & \left\langle\left(\left(d v^{t}\right)^{*} \nabla^{\prime} \nabla_{\bar{t}}+R^{*}\right)\left(\left(d v^{l}\right)^{*} \nabla^{\prime} \nabla_{\bar{l}}+R^{*}\right)\left(\left(d v^{s}\right)^{*} \nabla^{\prime} \nabla_{\bar{s}}+R^{*}\right) i_{\mu} u, i_{\mu} u\right\rangle \\
& =O(k)+\left\langle\left(d v^{t}\right)^{*} \nabla^{\prime} \nabla_{\bar{t}}\left(d v^{l}\right)^{*} \nabla^{\prime} \nabla_{\bar{l}}\left(d v^{s}\right)^{*} \nabla^{\prime} \nabla_{\bar{s}} i_{\mu} u, i_{\mu} u\right\rangle \\
& =O(k)+\left\langle\left(d v^{t}\right)^{*} \nabla^{\prime}\left(d v^{l}\right)^{*} \nabla_{\bar{t}} \nabla^{\prime}\left(d v^{s}\right)^{*} \nabla_{\bar{l}} \nabla^{\prime} \nabla_{\bar{s}} i_{\mu} u, i_{\mu} u\right\rangle \\
& =O(k)+k^{2}\left\langle\left(d v^{t}\right)^{*} \nabla^{\prime}\left(d v^{l}\right)^{*} \partial \phi_{\bar{t}} \wedge\left(d v^{s}\right)^{*} \partial \phi_{\bar{l}} \wedge \nabla_{\bar{s}} i_{\mu} u, i_{\mu} u\right\rangle \\
& =O(k)+k^{2}\left\langle\partial \phi_{\bar{t}} \wedge\left(d v^{s}\right)^{*} \partial \phi_{\bar{l}} \wedge \nabla_{\bar{s}} i_{\mu} u, d v^{l} \wedge \nabla^{*}\left(d v^{t} \wedge i_{\mu} u\right)\right\rangle \\
& =O(k)+k^{2}\left\langle-\phi_{i \bar{l}}\left(\nabla_{\bar{t}} \mu_{\bar{j}}^{i}\right) d \bar{v}^{j} u, \bar{\partial}\left(\phi^{l \bar{m}} \mu_{\bar{m}}^{t}\right) u\right\rangle \\
& =\int_{\mathcal{X}_{y}}\left(-k^{2}|\bar{\nabla} \mu|^{2}+O(k)\right)|u|^{2} e^{-k \phi},
\end{aligned}
$$

where the second equality holds because all the terms in

$$
\left\langle\left(\left(d v^{t}\right)^{*} \nabla^{\prime} \nabla_{\bar{t}}+R^{*}\right)\left(\left(d v^{l}\right)^{*} \nabla^{\prime} \nabla_{\bar{l}}+R^{*}\right)\left(\left(d v^{s}\right)^{*} \nabla^{\prime} \nabla_{\bar{s}}+R^{*}\right) i_{\mu} u, i_{\mu} u\right\rangle
$$

containing the factor $R^{*}$ are treated similarly as $\left\langle\left(k-\Delta^{\prime}\right)^{2} i_{\mu} u, i_{\mu} u\right\rangle$, which are in $O(k)$, the third equality holds since $\left[\nabla_{\bar{t}},\left(d v^{l}\right)^{*}\right]=\partial_{\bar{t}}\left(\phi^{\overline{l i}}\right) i_{\frac{\partial}{\partial v^{i}}}$, and so its adjoint operator is in $O(1)$, the fourth equality follows from $\left[\nabla_{\bar{t}}, \nabla^{\prime}\right]=k \partial \phi_{\bar{t}}$.

Lemma 3.6 The following expansion holds

$$
\left\langle\left(k-\Delta^{\prime}\right)^{4} i_{\mu} u, i_{\mu} u\right\rangle=\int_{\mathcal{X}_{y}}\left(k^{3}|\bar{\nabla} \mu|^{2}+O\left(k^{2}\right)\right)|u|^{2} e^{-k \phi} .
$$

Proof Similar to the proof in Lemma 3.5 for estimating reminder terms we have

$$
\begin{aligned}
& \left\langle\left(k-\Delta^{\prime}\right)^{4} i_{\mu} u, i_{\mu} u\right\rangle \\
& =\left\langle\left(\left(d v^{q}\right)^{*} \nabla^{\prime} \nabla_{\bar{q}}+R^{*}\right)\left(\left(d v^{t}\right)^{*} \nabla^{\prime} \nabla_{\bar{t}}+R^{*}\right)\left(\left(d v^{l}\right)^{*} \nabla^{\prime} \nabla_{\bar{l}}+R^{*}\right)\left(\left(d v^{s}\right)^{*} \nabla^{\prime} \nabla_{\bar{s}}+R^{*}\right) i_{\mu} u, i_{\mu} u\right\rangle \\
& =O\left(k^{2}\right)+\left\langle\left(d v^{q}\right)^{*} \nabla^{\prime} \nabla_{\bar{q}}\left(d v^{t}\right)^{*} \nabla^{\prime} \nabla_{\bar{t}}\left(d v^{l}\right)^{*} \nabla^{\prime} \nabla_{\bar{l}}\left(d v^{s}\right)^{*} \nabla^{\prime} \nabla_{\bar{s}} i_{\mu} u, i_{\mu} u\right\rangle \\
& =O\left(k^{2}\right)+\left\langle\left(d v^{q}\right)^{*} \nabla^{\prime}\left(d v^{t}\right)^{*} \nabla_{\bar{q}} \nabla^{\prime}\left(d v^{l}\right)^{*} \nabla_{\bar{t}} \nabla^{\prime}\left(d v^{s}\right)^{*} \nabla_{\bar{l}} \nabla^{\prime} \nabla_{\bar{s}} i_{\mu} u, i_{\mu} u\right\rangle \\
& =O\left(k^{2}\right)-k^{3}\left\langle\left(d v^{q}\right)^{*} \nabla^{\prime}\left(d v^{t}\right)^{*} \partial \phi_{\bar{q}} \wedge\left(d v^{l}\right)^{*} \partial \phi_{\bar{t}} \wedge\left(d v^{s}\right)^{*} \partial \phi_{\bar{l}} \wedge \nabla_{\bar{s}} i_{\mu} u, i_{\mu} u\right\rangle \\
& =O\left(k^{2}\right)-k^{3}\left\langle\partial \phi_{\bar{l}} \wedge \nabla_{\bar{s}} i_{\mu} u, d v^{s} \wedge \nabla^{*}\left(d v^{l} \wedge i_{\mu} u\right)\right\rangle \\
& =O\left(k^{2}\right)+k^{3}\left\langle\phi_{\bar{i}}\left(\nabla_{\bar{s}} \mu_{\bar{j}}^{i}\right) d \bar{v}^{j} u, \bar{\partial}\left(\phi^{s \bar{m}} \mu_{\bar{m}}^{l}\right) u\right\rangle \\
& =\int_{\mathcal{X}_{y}}\left(k^{3}|\bar{\nabla} \mu|^{2}+O\left(k^{2}\right)\right)|u|^{2} e^{-k \phi} .
\end{aligned}
$$

We prove now Theorem 1.1. 
Proof The curvature formula in (2.7) contains two quadratic forms in $u$, the first one invovles only $|u|^{2}$ and will be treated using Bergman kernel expansion later, the second one involves $\left(\Delta^{\prime}+k\right)^{-1}$ and will be treated first. We have, by the expansion (3.4)

$$
\begin{gathered}
\left\langle\left(\Delta^{\prime}+k\right)^{-1} i_{\mu} u, i_{\mu} u\right\rangle=I(u)+I I(u)+\cdots+V I I(u) \\
I(u)=\left\langle I i_{\mu} u, i_{\mu} u\right\rangle, \quad I I(u)=\left\langle I I i_{\mu} u, i_{\mu} u\right\rangle, \cdots, V I I(u)=\left\langle V I I i_{\mu} u, i_{\mu} u\right\rangle .
\end{gathered}
$$

We shall treat each term using the lemmas above. First we have

$$
I(u)=\left\langle\frac{1}{2 k} i_{\mu} u, i_{\mu} u\right\rangle=\frac{1}{2 k} \int_{\mathcal{X}_{y}}\left(\mu_{\bar{j}}^{i} \overline{\mu_{\bar{t}}^{s}} \phi_{i \bar{s}} \phi^{\bar{j} t}\right)|u|^{2} e^{-\phi}=\frac{1}{2 k} \int_{\mathcal{X}_{y}}|\mu|^{2}|u|^{2} e^{-k \phi},
$$

where $|\mu|^{2}=\mu_{\bar{j}}^{i} \overline{\mu_{\bar{t}}^{s}} \phi_{i \bar{s}} \phi^{\bar{j} t}$.

By Lemma 3.3 and (3.11), the second term is

$$
I I(u)=-\frac{1}{6 k^{2}} \int_{\mathcal{X}_{y}}|\bar{\nabla} \mu|^{2}|u|^{2} e^{-k \phi} .
$$

Likewise, by (3.11)

$$
I I I(u)=\frac{1}{4 k^{2}} \int_{\mathcal{X}_{y}}|\mu|_{R^{*}}^{2}|u|^{2} e^{-k \phi} .
$$

The fourth term is

$$
\begin{aligned}
I V(u) & \leq \frac{1}{4 k^{2}}\left\|R^{*} i_{\mu} u\right\| \cdot\left\|\left(\Delta^{\prime}+k\right)^{-1}\left(k-\Delta^{\prime}\right) i_{\mu} u\right\| \\
& \leq \frac{1}{4 k^{3}}\left\|R^{*} i_{\mu} u\right\| \cdot\left\langle\left(k-\Delta^{\prime}\right)^{2} i_{\mu} u, i_{\mu} u\right\rangle^{\frac{1}{2}} \\
& =\frac{1}{4 k^{3}}(O(1)\|\mu\|) \cdot\left(O\left(k^{\frac{1}{2}}\right)\|u\|\right) \\
& =O\left(\frac{1}{k^{\frac{5}{2}}}\right)\|u\|^{2},
\end{aligned}
$$

where the third equality follows from Lemma 3.4.

By Lemmas 3.2, 3.3 and 3.4, the fifth term

$$
\begin{aligned}
V(u)= & \frac{1}{18 k^{2}}\left\langle\left(k-\Delta^{\prime}-R^{*}\right) i_{\mu} u, i_{\mu} u\right\rangle+\frac{1}{18 k^{3}}\left(\left\langle\left(k-\Delta^{\prime}\right)^{2} i_{\mu} u, i_{\mu} u\right\rangle\right. \\
& \left.-\left\langle\left(k-\Delta^{\prime}\right) R^{*} i_{\mu} u, i_{\mu} u\right\rangle\right) \\
= & -\frac{1}{18 k^{2}} \int_{\mathcal{X}_{y}}|\bar{\nabla} \mu|^{2}|u|^{2} e^{-k \phi}+\frac{1}{18 k^{3}} \int_{\mathcal{X}_{y}}\left(k|\bar{\nabla} \mu|^{2}+O(1)\right)|u|^{2} e^{-k \phi} \\
- & \frac{1}{18 k^{3}}\left(\left\langle\left(R^{*}\right)^{2} i_{\mu} u, i_{\mu} u\right\rangle+\left\langle\nabla_{\frac{\partial}{\partial \bar{v}^{t}}} R^{*} i_{\mu} u, \nabla^{\prime *}\left(d v^{t} \wedge i_{\mu} u\right)\right\rangle\right) \\
= & O\left(\frac{1}{k^{3}}\right)\|u\|^{2} .
\end{aligned}
$$


By (3.20), (3.23), Lemmas 3.5 and 3.4, the sixth term becomes

$$
\begin{aligned}
V I(u)= & \left\langle\frac{1}{36 k^{4}}\left(k^{2}+2 k(k-\Delta)+\left(k-\Delta^{\prime}\right)^{2}\right)\left(k-\Delta^{\prime}-R^{*}\right) i_{\mu} u, i_{\mu} u\right\rangle \\
= & \frac{1}{36 k^{2}}\left\langle\left(k-\Delta^{\prime}-R^{*}\right) i_{\mu} u, i_{\mu} u\right\rangle+\frac{1}{18 k^{3}}\left\langle\left(k-\Delta^{\prime}\right)\left(k-\Delta^{\prime}-R^{*}\right) i_{\mu} u, i_{\mu} u\right\rangle \\
& +\frac{1}{36 k^{4}}\left\langle\left(k-\Delta^{\prime}\right)^{3} i_{\mu} u, i_{\mu} u\right\rangle-\frac{1}{36 k^{4}}\left\langle\left(k-\Delta^{\prime}\right)^{2} R^{*} i_{\mu} u, i_{\mu} u\right\rangle \\
= & -\frac{1}{36 k^{2}}\left\langle\left(k-\Delta^{\prime}-R^{*}\right) i_{\mu} u, i_{\mu} u\right\rangle+\frac{1}{18 k^{3}}\left\langle\left(2 k-\Delta^{\prime}\right)\left(k-\Delta^{\prime}-R^{*}\right) i_{\mu} u, i_{\mu} u\right\rangle \\
& +\frac{1}{36 k^{4}}\left\langle\left(k-\Delta^{\prime}\right)^{3} i_{\mu} u, i_{\mu} u\right\rangle-\frac{1}{36 k^{4}}\left\langle\left(k-\Delta^{\prime}\right)^{2} R^{*} i_{\mu} u, i_{\mu} u\right\rangle \\
= & O\left(\frac{1}{k^{3}}\right)\|u\|^{2} .
\end{aligned}
$$

Here we have used Lemmas 3.3 and 3.5 to conclude

$$
\begin{aligned}
- & \frac{1}{36 k^{2}}\left\langle\left(k-\Delta^{\prime}-R^{*}\right) i_{\mu} u, i_{\mu} u\right\rangle+\frac{1}{36 k^{4}}\left\langle\left(k-\Delta^{\prime}\right)^{3} i_{\mu} u, i_{\mu} u\right\rangle \\
= & -\frac{1}{36 k^{2}} \int_{\mathcal{X}_{y}}\left(-|\bar{\nabla} \mu|^{2}\right)|u|^{2} e^{-k \phi}+\frac{1}{36 k^{4}} \int_{\mathcal{X}_{y}}\left(-k^{2}|\bar{\nabla} u|^{2}+O(k)\right)|u|^{2} e^{-k \phi} \\
& =\frac{1}{36 k^{4}} \int_{\mathcal{X}_{y}} O(k)|u|^{2} e^{-k \phi}=O\left(\frac{1}{k^{3}}\right)\|u\|^{2} .
\end{aligned}
$$

Finally the last term is

$$
\begin{aligned}
V I I(u)= & \frac{1}{36 k^{4}}\left\langle\left(\Delta^{\prime}+k\right)^{-1}\left(k-\Delta^{\prime}\right)\left(2 k-\Delta^{\prime}\right) i_{\mu} u,\left(k-\Delta^{\prime}\right)\left(2 k-\Delta^{\prime}\right) i_{\mu} u\right\rangle \\
& -\frac{1}{36 k^{4}}\left\langle\left(2 k-\Delta^{\prime}\right)^{2} R^{*} i_{\mu} u,\left(\Delta^{\prime}+k\right)^{-1}\left(k-\Delta^{\prime}\right) i_{\mu} u\right\rangle .
\end{aligned}
$$

Note that

$$
\begin{aligned}
& \left\langle\frac{1}{36 k^{4}}\left(\Delta^{\prime}+k\right)^{-1}\left(k-\Delta^{\prime}\right)\left(2 k-\Delta^{\prime}\right) i_{\mu} u,\left(k-\Delta^{\prime}\right)\left(2 k-\Delta^{\prime}\right) i_{\mu} u\right\rangle \\
& \leq \frac{1}{36 k^{5}}\left\langle\left(k-\Delta^{\prime}\right)\left(2 k-\Delta^{\prime}\right) i_{\mu} u,\left(k-\Delta^{\prime}\right)\left(2 k-\Delta^{\prime}\right) i_{\mu} u\right\rangle \\
& =\frac{1}{36 k^{5}}\left\langle\left(k-\Delta^{\prime}\right)^{4} i_{\mu} u, i_{\mu} u\right\rangle+\frac{1}{18 k^{4}}\left\langle\left(k-\Delta^{\prime}\right)^{3} i_{\mu} u, i_{\mu} u\right\rangle \\
& \quad+\frac{1}{36 k^{3}}\left\langle\left(k-\Delta^{\prime}\right)^{2} i_{\mu} u, i_{\mu} u\right\rangle=O\left(\frac{1}{k^{3}}\right)\|u\|^{2},
\end{aligned}
$$

where the last equality follows from Lemmas 3.4, 3.5 and 3.6. 
We estimate the second term in the RHS of (3.26) as

$$
\begin{aligned}
\mid- & \frac{1}{36 k^{4}}\left\langle\left(2 k-\Delta^{\prime}\right)^{2} R^{*} i_{\mu} u,\left(\Delta^{\prime}+k\right)^{-1}\left(k-\Delta^{\prime}\right) i_{\mu} u\right\rangle \mid \\
& \leq \frac{1}{36 k^{4}}\left\|\left(2 k-\Delta^{\prime}\right)^{2} R^{*} i_{\mu} u\right\| \cdot\left\|\left(\Delta^{\prime}+k\right)^{-1}\left(k-\Delta^{\prime}\right) i_{\mu} u\right\| \\
& \leq \frac{1}{36 k^{5}}\left\|\left(2 k-\Delta^{\prime}\right)^{2} R^{*} i_{\mu} u\right\| \cdot\left\langle\left(k-\Delta^{\prime}\right)^{2} i_{\mu} u, i_{\mu} u\right\rangle^{1 / 2} \\
& =\frac{1}{36 k^{5}}\left(O\left(k^{2}\right)\|u\|\right) \cdot\left(O\left(k^{\frac{1}{2}}\right)\|u\|\right) \\
& =O\left(\frac{1}{k^{\frac{5}{2}}}\right)\|u\|^{2}
\end{aligned}
$$

indeed the third equality follows from Lemma 3.4 and the following equality

$$
\begin{aligned}
& \left\|\left(2 k-\Delta^{\prime}\right)^{2} R^{*} i_{\mu} u\right\|=\left|\left\langle\left(2 k-\Delta^{\prime}\right)^{4} R^{*} i_{\mu} u, R^{*} i_{\mu} u\right\rangle\right|^{1 / 2} \\
& \quad=\left|\left\langle\left(k^{4}+4 k^{3}\left(k-\Delta^{\prime}\right)+6 k^{2}\left(k-\Delta^{\prime}\right)^{2}+4 k\left(k-\Delta^{\prime}\right)^{3}+\left(k-\Delta^{\prime}\right)^{4}\right) i_{\tilde{\mu}} u, i_{\tilde{\mu}} u\right\rangle\right|^{1 / 2} \\
& \quad=O\left(k^{2}\right)\|u\|,
\end{aligned}
$$

where the last equality is obtained from Lemmas 3.3,3.4, 3.5 and 3.6, with $\tilde{\mu}=R_{i \bar{l}}^{\bar{t} j} \mu_{\bar{t}}^{i} d \bar{v}^{l} \otimes$ $\frac{\partial}{\partial v^{j}}$ being the contraction of $\mu$ agains the curvature tensor $R$.

Substituting (3.26) and (3.27) into (3.25) results in

$$
\left\langle\frac{1}{36 k^{4}}\left(\Delta^{\prime}+k\right)^{-1}\left(k-\Delta^{\prime}\right)\left(2 k-\Delta^{\prime}\right)^{2}\left(k-\Delta^{\prime}-R^{*}\right) i_{\mu} u, i_{\mu} u\right\rangle=O\left(\frac{1}{k^{\frac{5}{2}}}\right)\|u\|^{2} .
$$

Putting the quantities (3.19), (3.20), (3.21), (3.22), (3.23), (3.24), (3.29) into (3.4), we obtain

$$
\begin{aligned}
& \left\langle\left(\Delta^{\prime}+k\right)^{-1} i_{\mu} u, i_{\mu} u\right\rangle \\
& \quad=\int_{\mathcal{X}_{y}}\left(\frac{1}{2 k}|\mu|^{2}+\left(-\frac{1}{6}|\bar{\nabla} \mu|^{2}+\frac{1}{4}|\mu|_{R^{*}}^{2}\right) \frac{1}{k^{2}}+o\left(k^{-2}\right)\right)|u|^{2} e^{-k \phi} .
\end{aligned}
$$

Finally substituting (3.30) into (3.2) we get

$$
\begin{aligned}
& \left\langle\Theta^{E^{k}} u, u\right\rangle(\zeta, \bar{\zeta})=-\sqrt{-1}\left(\int_{\mathcal{X}_{y}} k c(\phi)|u|^{2} e^{-k \phi}\right)(\zeta, \bar{\zeta}) \\
& \quad+\int_{\mathcal{X}_{y}}\left(\frac{1}{2}|\mu|^{2}+\left(-\frac{1}{6}|\bar{\nabla} \mu|^{2}+\frac{1}{4}|\mu|_{R^{*}}^{2}\right) \frac{1}{k}+o\left(k^{-1}\right)\right)|u|^{2} e^{-k \phi} .
\end{aligned}
$$

Denote $d_{k}=\operatorname{dim} H^{0}\left(\mathcal{X}_{y}, L^{k}+K_{\mathcal{X} / M}\right)$, and let $\left\{u_{j}\right\}_{j=1}^{d_{k}}$ be an orthogonal basis of $H^{0}\left(\mathcal{X}_{y}, L^{k}+K_{\mathcal{X} / M}\right)$. From (2.12) and (3.56), we have

$$
\begin{aligned}
& \sum_{j=1}^{d_{k}}\left|u_{j}\right|^{2} e^{-k \phi}=\sum_{j=1}^{d_{k}}\left|u_{j}\right|_{L^{2}}^{2} \frac{\omega^{n}}{n !} \\
& \quad=\left(k^{n}-\frac{\rho}{2} k^{n-1}+\left(-\frac{1}{6} \Delta \rho+\frac{1}{24}\left(|R|^{2}-4|R i c|^{2}+3 \rho^{2}\right)\right) k^{n-2}+o\left(k^{n-2}\right)\right) \frac{c(L, \phi)^{n}}{n !},
\end{aligned}
$$


where $c(L, \phi)=\frac{1}{2 \pi} \omega$ is the Chern form of the Hermitian line bundle $\left(L, e^{-\phi}\right)$.

We take the trace to both sides of (3.31) and use the Bergman kernel expansion (3.32),

$$
\begin{aligned}
- & \sqrt{-1} c_{1}\left(E^{k},\|\bullet\|_{k}\right)(\zeta, \bar{\zeta})=\frac{1}{2 \pi} \operatorname{tr} \Theta^{E_{k}}(\zeta, \bar{\zeta}) \\
= & \frac{1}{2 \pi} \int_{\mathcal{X}_{y}}\left(-\sqrt{-1} k c(\phi)(\zeta, \bar{\zeta})+\frac{1}{2}|\mu|^{2}+\left(-\frac{1}{6}|\bar{\nabla} \mu|^{2}+\frac{1}{4}|\mu|_{R^{*}}^{2}\right) \frac{1}{k}+o\left(k^{-1}\right)\right) \\
& \cdot\left(k^{n}-\frac{\rho}{2} k^{n-1}+\left(-\frac{1}{6} \Delta \rho+\frac{1}{24}\left(|R|^{2}-4|R i c|^{2}+3 \rho^{2}\right)\right) k^{n-2}+o\left(k^{n-2}\right)\right) \frac{c(L, \phi)^{n}}{n !} \\
= & \frac{k^{n+1}}{(2 \pi)^{n+1}} \int_{\mathcal{X}_{y}}(-\sqrt{-1}) c(\phi)(\zeta, \bar{\zeta}) \frac{\omega^{n}}{n !}+\frac{k^{n}}{(2 \pi)^{n+1}} \int_{\mathcal{X}_{y}}\left(\frac{1}{2}|\mu|^{2}-\frac{\rho}{2}(-\sqrt{-1}) c(\phi)(\zeta, \bar{\zeta})\right) \frac{\omega^{n}}{n !} \\
& +\frac{k^{n-1}}{(2 \pi)^{n+1}} \int_{\mathcal{X}_{y}}\left((-\sqrt{-1}) c(\phi)(\zeta, \bar{\zeta})\left(-\frac{1}{6} \Delta \rho+\frac{1}{24}\left(|R|^{2}-4|R i c|^{2}+3 \rho^{2}\right)\right)-\frac{\rho}{4}|\mu|^{2}\right) \frac{\omega^{n}}{n !} \\
& +\frac{k^{n-1}}{(2 \pi)^{n+1}}\left(-\frac{1}{6}\|\bar{\nabla} \mu\|^{2}+\frac{1}{4}\|\mu\|_{R^{*}}^{2}\right)+o\left(k^{n-1}\right),
\end{aligned}
$$

where we have denoted $\|\bar{\nabla} \mu\|^{2}=\int_{\mathcal{X}_{y}}|\bar{\nabla} \mu|^{2} \frac{\omega^{n}}{n !},\|\mu\|_{R^{*}}^{2}=\int_{\mathcal{X}_{y}}|\mu|_{R^{*}}^{2} \frac{\omega^{n}}{n !}$. We rewrite the integrals $\|\mu\|_{R^{*}}^{2}$ and $\|\bar{\nabla} \mu\|^{2}$ (of the anti-holomorphic connection $\bar{\nabla} \mu$ ) in terms of $\partial^{*} \mu$ and the holomorphic connection $\nabla^{\prime} \mu$. By Akizuki-Nakano identity [15, Chapter VII, Corollary (1.3)], we have

$$
\begin{aligned}
\left\|\bar{\partial}^{*} \mu\right\|^{2} & =\left\langle\Delta^{\prime \prime} \mu, \mu\right\rangle \\
& =\left\langle\Delta^{\prime} \mu, \mu\right\rangle+\langle[\sqrt{-1} R, \Lambda] \mu, \mu\rangle \\
& =\left\|\nabla^{\prime} \mu\right\|^{2}-\langle\sqrt{-1} \Lambda R \mu, \mu\rangle \\
& =\left\|\nabla^{\prime} \mu\right\|^{2}-\left\langle\left(\phi^{\bar{s} t} \mu_{\bar{j}}^{i} R_{i s \bar{t}}^{k} d \bar{v}^{j}-\phi^{s \bar{t}} \mu_{\bar{t}}^{i} R_{i s \bar{j}}^{k} d \bar{v}^{j}\right) \otimes \frac{\partial}{\partial v^{k}}, \mu\right\rangle \\
& =\left\|\nabla^{\prime} \mu\right\|^{2}-\left\langle\left(\mu_{\bar{j}}^{i} R_{i \bar{l}} \phi^{\bar{l} k} d \bar{v}^{j}-\phi^{s \bar{t}} \mu_{\bar{t}}^{i} R_{i s \bar{j}}^{k} d \bar{v}^{j}\right) \otimes \frac{\partial}{\partial v^{k}}, \mu\right\rangle \\
& =\left\|\nabla^{\prime} \mu\right\|^{2}-\int_{\mathcal{X}_{y}}\left(\mu_{\bar{j}}^{i} \overline{\mu_{\bar{t}}^{s}} R_{i \bar{l}} \phi^{\bar{l} k} \phi^{\bar{j} t} \phi_{k \bar{s}}\right) \frac{\omega^{n}}{n !}+\int_{\mathcal{X}_{y}} \phi^{s \bar{t}} R_{i s \bar{j}}^{k} \mu_{\bar{t}}^{i} \mu_{\bar{q}}^{p} \phi^{\bar{j} q} \phi_{k \bar{p}} \frac{\omega^{n}}{n !} \\
& =\left\|\nabla^{\prime} \mu\right\|^{2}-\|\mu\|_{R i c}^{2}-\|\mu\|_{R^{*}}^{2},
\end{aligned}
$$

where we have denoted $\|\mu\|_{R i c}^{2}:=\int_{\mathcal{X}_{y}}\left(\mu_{\bar{j}}^{i} \overline{\mu_{\bar{t}}^{s}} R_{i \bar{l}} \phi^{\bar{l} k} \phi^{\bar{j} t} \phi_{k \bar{s}}\right) \frac{\omega^{n}}{n !}$, which again needs not to be nonnegative. Therefore,

$$
\|\mu\|_{R^{*}}^{2}=\left\|\nabla^{\prime} \mu\right\|^{2}-\|\mu\|_{R i c}^{2}-\left\|\bar{\partial}^{*} \mu\right\|^{2} .
$$


By its definition we find also

$$
\begin{aligned}
\|\bar{\nabla} \mu\|^{2} & =\int_{\mathcal{X}_{y}} \nabla_{\bar{l}} \mu_{\bar{j}}^{i} \phi^{\bar{l} k} \nabla_{k} \overline{\mu_{\bar{i}}^{j}} \frac{\omega^{n}}{n !}=-\int_{\mathcal{X}_{y}} \nabla_{k} \nabla_{\bar{l}} \mu_{\bar{j}}^{i} \phi^{\bar{l} k} \overline{\mu_{\bar{i}}^{j}} \frac{\omega^{n}}{n !} \\
& =\int_{\mathcal{X}_{y}}\left(\nabla_{\bar{l}} \nabla_{k}-\nabla_{k} \nabla_{\bar{l}}\right) \mu_{\bar{j}}^{i} \phi^{\bar{l} k} \overline{\mu_{\bar{i}}^{j}} \frac{\omega^{n}}{n !}-\int_{\mathcal{X}_{y}} \nabla_{\bar{l}} \nabla_{k} \mu_{\bar{j}}^{i} \phi^{\bar{l} k} \overline{\mu_{\bar{i}}^{j}} \frac{\omega^{n}}{n !} \\
& =\int_{\mathcal{X}_{y}}\left(\partial_{\bar{l}} \Gamma_{k s}^{i} \mu_{\bar{j}}^{s}+\partial_{k} \overline{\Gamma_{l j}^{s}} \mu_{\bar{s}}^{i}\right) \phi^{\bar{l} k} \overline{\mu_{\bar{i}}^{j}} \frac{\omega^{n}}{n !}+\int_{\mathcal{X}_{y}} \nabla_{k} \mu_{\bar{j}}^{i} \phi^{\bar{l} k} \overline{\nabla_{l}} \mu_{\bar{i}}^{j} \frac{\omega^{n}}{n !} \\
& =-2\|\mu\|_{R i c}^{2}+\left\|\nabla^{\prime} \mu\right\|^{2} .
\end{aligned}
$$

Substituting (3.34) and (3.35) into (3.33) we obtain finally

$$
\begin{aligned}
- & \sqrt{-1} c_{1}\left(E^{k},\|\bullet\|_{k}\right)(\zeta, \bar{\zeta})=\frac{k^{n+1}}{(2 \pi)^{n}} \\
& \times \int_{\mathcal{X}_{y}}(-\sqrt{-1}) c(\phi)(\zeta, \bar{\zeta}) \frac{\omega^{n}}{n !}+\frac{k^{n}}{(2 \pi)^{n}} \int_{\mathcal{X}_{y}}\left(\frac{1}{2}|\mu|^{2}-\frac{\rho}{2}(-\sqrt{-1}) c(\phi)(\zeta, \bar{\zeta})\right) \frac{\omega^{n}}{n !} \\
& +\frac{k^{n-1}}{(2 \pi)^{n+1}} \int_{\mathcal{X}_{y}}\left((-\sqrt{-1}) c(\phi)(\zeta, \bar{\zeta})\left(-\frac{1}{6} \Delta \rho+\frac{1}{24}\left(|R|^{2}-4|R i c|^{2}+3 \rho^{2}\right)\right)-\frac{\rho}{4}|\mu|^{2}\right) \frac{\omega^{n}}{n !} \\
& +\frac{k^{n-1}}{(2 \pi)^{n+1}}\left(\frac{1}{12}\|\mu\|_{R i c}^{2}+\frac{1}{12}\left\|\nabla^{\prime} \mu\right\|^{2}-\frac{1}{4}\left\|\bar{\partial}^{*} \mu\right\|^{2}\right)+o\left(k^{n-1}\right) .
\end{aligned}
$$

This completes the proof of Theorem 1.1.

Remark 3.7 It is a general fact [22] that the $L^{2}$-curvature $c_{1}\left(E^{k}\right)$ above has an expansion in the integer powers of $k$, so that the lower order term $o\left(k^{n-1}\right)$ in our statement can be written as $O\left(k^{n-2}\right)$. Indeed observe that the fractional order $O\left(k^{n-1-\frac{1}{2}}\right)$-terms in the proof of Theorem 1.1 are all due to the estimate $\left\|\left(\Delta^{\prime}+k\right)^{-1}\left(k-\Delta^{\prime}\right) i_{\mu} u\right\| \leq C k^{-\frac{1}{2}}\|u\|$. However we can use again Lemma 3.1 and prove that the traces of the quadratic forms involving $\left(\Delta^{\prime}+k\right)^{-1}\left(k-\Delta^{\prime}\right)$ are actually of integer order instead of fractional order, e.g. the trace of the quadratic form $I V(u)$ in the estimate (3.22) has an expansion of order $k^{-3+n}$. It might be interesting to find a recursive formula for the coefficients of the expansion $c_{1}\left(E^{k}\right)$ following our proof and using the Bergman kernel expansion.

\subsection{The asymptotic of the curvature of Quillen metric}

We compute now the asymptotic of the curvature of Qullien metric and prove Theorem 1.2. 
By Theorems 2.7 and 2.8, we have

$$
\begin{aligned}
- & \left.c_{1}\left(\lambda,\|\bullet\|_{Q}\right)=\left\{\int_{\mathcal{X} / M} \operatorname{td}\left(\mathcal{X} / M,(\sqrt{-1} \partial \bar{\partial} \phi)_{y}\right)\right) e^{\frac{1}{2 \pi}\left(k \omega+\sqrt{-1} R^{K} \mathcal{X} / M\right)}\right\}^{(1,1)} \\
= & \left\{\int_{\mathcal{X} / M}\left(1+\frac{1}{2} c_{1}\left(T_{\mathcal{X} / M}, \phi\right)+\frac{1}{12}\left(c_{1}\left(T_{\mathcal{X} / M}, \phi\right)^{2}+c_{2}\left(T_{\mathcal{X} / M}, \phi\right)\right)+\cdots\right)\right. \\
& \left.\cdot \sum_{i=0}^{\infty} \frac{(2 \pi)^{-i}\left(k \omega+\sqrt{-1} R^{K \mathcal{X} / M}\right)^{i}}{i !}\right\}^{(1,1)} \\
= & \frac{k^{n+1}}{(2 \pi)^{n+1}} \int_{\mathcal{X} / M} \frac{\omega^{n+1}}{(n+1) !}+\frac{k^{n}}{(2 \pi)^{n+1}} \int_{\mathcal{X} / M} \frac{1}{2}\left(\sqrt{-1} R^{K} \mathcal{X} / M\right) \wedge \frac{\omega^{n}}{n !} \\
& +\frac{k^{n-1}}{(2 \pi)^{n+1}} \int_{\mathcal{X} / M} \frac{1}{12}\left(\sqrt{-1} R^{K} \mathcal{X} / M\right)^{2} \wedge \frac{\omega^{n-1}}{(n-1) !} \\
& +\frac{k^{n-1}}{(2 \pi)^{n-1}} \int_{\mathcal{X} / M} \frac{1}{12} c_{2}\left(T_{\mathcal{X} / M}, \phi\right) \wedge \frac{\omega^{n-1}}{(n-1) !}+O\left(k^{n-2}\right),
\end{aligned}
$$

where td is the Todd character forms, which has an expansion,

$$
\operatorname{td}(F, h)=1+\frac{1}{2} c_{1}(F, h)+\frac{1}{12}\left(c_{1}(F, h)^{2}+c_{2}(F, h)\right)+\frac{1}{24} c_{1}(F, h) c_{2}(F, h)+\cdots
$$

for any Hermitian vector bundle $(F, h)$, the second equality follows from $c_{1}\left(T_{\mathcal{X} / M}, \phi\right)=$ $-\frac{\sqrt{-1}}{2 \pi} \partial \bar{\partial} \log \operatorname{det} \phi=-\frac{\sqrt{-1}}{2 \pi} R^{K \mathcal{X} / M}$.

Now we consider the last term in the RHS of (3.37),

$$
\begin{aligned}
& \int_{\mathcal{X} / M} c_{2}\left(T_{\mathcal{X} / M}, \phi\right) \wedge \frac{\omega^{n-1}}{12(n-1) !} \\
& =\int_{\mathcal{X} / M} \frac{1}{2}\left(c_{1}\left(K_{\mathcal{X} / M}, \phi\right)^{2}-\left(\frac{\sqrt{-1}}{2 \pi}\right)^{2} \operatorname{tr}(R \wedge R)\right) \wedge \frac{\omega^{n-1}}{12(n-1) !} \\
& =\left(\frac{1}{2 \pi}\right)^{2} \int_{\mathcal{X} / M} \frac{1}{24}\left(\sqrt{-1} R^{K} \mathcal{X} / M\right)^{2} \wedge \frac{\omega^{n-1}}{(n-1) !} \\
& \quad-\left(\frac{1}{2 \pi}\right)^{2} \int_{\mathcal{X} / M} \frac{1}{24}(\sqrt{-1})^{2} \operatorname{tr}(R \wedge R) \wedge \frac{\omega^{n-1}}{(n-1) !},
\end{aligned}
$$

where the curvature operator $R$ is defined by

$$
\begin{aligned}
R & =R_{j}^{i} \delta v^{j} \otimes i_{\frac{\partial}{\partial v^{i}}} \\
& =\left(R_{j \alpha \bar{\beta}}^{i} d z^{\alpha} \wedge d \bar{z}^{\beta}+R_{j \alpha \bar{\beta}}^{i} d z^{\alpha} \wedge \delta \bar{v}^{l}+R_{j k \bar{\beta}}^{i} \delta v^{k} \wedge d \bar{z}^{\beta}+R_{j k \bar{l}}^{i} \delta v^{k} \wedge \delta \bar{v}^{l}\right) \delta v^{j} \otimes i_{\frac{\partial}{\partial v^{i}}} .
\end{aligned}
$$

Here the second and third curvature term is

$$
R_{j \alpha \bar{l}}^{i}=\nabla_{j}^{\prime}\left(\mu_{\alpha}\right)_{\bar{l}}^{i}, \quad R_{i k \bar{\beta}}^{j}=\overline{\nabla_{t}^{\prime}\left(\mu_{\beta}\right)_{\bar{k}}^{s}} \phi^{\bar{t} j} \phi_{\bar{s} i} .
$$


In fact, one can prove them in terms of normal coordinates, i.e. $\phi_{i \bar{j}}=\delta_{i j}, \phi_{i \bar{j} k}=0$ at a fix point, so

$$
\begin{aligned}
R_{i k \bar{\beta}}^{j} & =i_{\frac{\delta}{\delta \bar{z}^{\beta}}} i_{\frac{\partial}{\partial v^{k}}} \bar{\partial}\left(\partial \phi_{i \bar{l}} \phi^{\bar{l} j}\right) \\
& =-\left(\partial_{\bar{\beta}}-\phi_{\bar{\beta} t} \phi^{t \bar{s}} \partial_{\bar{s}}\right)\left(\phi_{i \bar{l} k} \phi^{\bar{l} j}\right) \\
& =-\phi_{i \bar{j} k \bar{\beta}}+\phi_{\bar{\beta} t} \phi_{i \bar{j} k \bar{t}} \\
& =\nabla_{j}^{\prime}\left(-\phi_{\alpha \bar{k} \bar{l}} \phi^{\bar{k} i}+\phi_{\alpha \bar{k}} \phi_{k \bar{i} \bar{l}}\right) \\
& =\nabla_{j}\left(-\partial_{\bar{l}}\left(\phi_{\alpha \bar{k}} \phi^{\bar{k} i}\right)\right)=\nabla_{j}^{\prime}\left(\mu_{\alpha}\right)_{\bar{l}}^{i},
\end{aligned}
$$

while second identity in (3.40) holds similarly.

We compute the second term in the RHS of (3.38),

$$
\begin{aligned}
(\sqrt{-1})^{2} \int_{\mathcal{X} / M} \operatorname{tr}(R \wedge R) \frac{\omega^{n-1}}{(n-1) !} \\
=\int_{\mathcal{X} / M}\left(2 R_{j \alpha \bar{\beta}}^{i} R_{i k \bar{l}}^{j} \phi^{k \bar{l}}-2 R_{j \alpha \bar{l}}^{i} R_{i k \bar{\beta}}^{j} \phi^{k \bar{l}}\right) \frac{\omega^{n}}{n !} \sqrt{-1} d z^{\alpha} \wedge d \bar{z}^{\beta} \\
\quad+\int_{\mathcal{X} / M} R_{j k \bar{l}}^{i} R_{i s \bar{t}}^{j} c(\phi)\left(\sqrt{-1} \delta v^{k} \wedge \delta \bar{v}^{l}\right) \wedge\left(\sqrt{-1} \delta v^{s} \wedge \delta \bar{v}^{t}\right) \wedge \frac{\omega^{n-1}}{(n-2) !} \\
=2 \int_{\mathcal{X} / M} R_{j \alpha \bar{\beta}}^{i} R_{i k \bar{l}}^{j} \phi^{k \bar{l}} \frac{\omega^{n}}{n !} \sqrt{-1} d z^{\alpha} \wedge d \bar{z}^{\beta}-2\left\langle\nabla^{\prime} \mu_{\alpha}, \nabla^{\prime} \mu_{\beta}\right\rangle \sqrt{-1} d z^{\alpha} \wedge d \bar{z}^{\beta} \\
\\
\quad+\int_{\mathcal{X} / M}\left(|R i c|^{2}-|R|^{2}\right) c(\phi) \frac{\omega^{n}}{n !},
\end{aligned}
$$

where the second equality follows from the fact

$$
n(n-1) \alpha \wedge \beta \wedge \omega^{n-2}=(\Lambda \alpha \cdot \Lambda \beta-\langle\alpha, \beta\rangle) \omega^{n}
$$

for two real $(1,1)$-forms $\alpha$ and $\beta$.

\section{Lemma 3.8 The following identities hold}

$$
\int_{\mathcal{X} / M}\left(\sqrt{-1} R^{K \mathcal{X} / M}\right) \wedge \frac{\omega^{n}}{n !}=-\int_{\mathcal{X} / M} \rho c(\phi) \frac{\omega^{n}}{n !}+\left\langle\mu_{\alpha}, \mu_{\beta}\right\rangle \sqrt{-1} d z^{\alpha} \wedge d \bar{z}^{\beta}
$$

and

$$
\int_{\mathcal{X} / M}(\Delta \rho) c(\phi) \frac{\omega^{n}}{n !}=\left(-\int_{\mathcal{X} / M}\left(R_{j \alpha \bar{\beta}}^{i} R_{i k \bar{l}}^{j} \phi^{k \bar{l}}\right) \frac{\omega^{n}}{n !}-\left\langle\mu_{\alpha}, \mu_{\beta}\right\rangle_{R i c}\right) \sqrt{-1} d z^{\alpha} \wedge d \bar{z}^{\beta},
$$

where $\left\langle\mu_{\alpha}, \mu_{\beta}\right\rangle_{R i c}:=\int_{\mathcal{X} / M}\left(\left(\mu_{\alpha}\right)_{\bar{j}}^{i} \overline{\left(\mu_{\beta}\right)_{\bar{t}}^{s}} R_{i \bar{l}} \phi^{\bar{l} k} \phi^{\bar{j} t} \phi_{k \bar{s}}\right) \frac{\omega^{n}}{n !}$, which satisfies

$$
\left\langle\mu_{\alpha}, \mu_{\beta}\right\rangle_{R i c} \zeta^{\alpha} \overline{\zeta^{\beta}}=\|\mu\|_{R i c}^{2}
$$

Proof For any fixed point $p \in \mathcal{X}_{y}, y \in M$, we take normal coordinates near $p$ such that $\phi_{i \bar{j}}(p)=\delta_{i j}, \phi_{i \bar{j} k}(p)=0$. Recall that $\left(\mu_{\alpha}\right)_{\bar{l}}^{k}=-\partial_{\bar{l}}\left(\phi_{\alpha \bar{t}} \phi^{\bar{t} k}\right)$ and denote $c(\phi)_{\alpha \bar{\beta}}=\phi_{\alpha \bar{\beta}}$ 
$\phi_{\alpha \bar{l}} \phi_{k \bar{\beta}} \phi^{k \bar{l}}$. Evaluating at $p$ we see that

$$
\begin{aligned}
\partial_{k} \partial_{\bar{l}} c(\phi)_{\alpha \bar{\beta}}= & \partial_{k} \partial_{\bar{l}}\left(\phi_{\alpha \bar{\beta}}-\phi_{\alpha \bar{j}} \phi^{\bar{j} i} \phi_{i \bar{\beta}}\right) \\
= & \phi_{k \bar{l} \alpha \bar{\beta}}-\phi_{\alpha \bar{j} k \bar{l}} \phi_{j \bar{\beta}}-\phi_{\alpha \bar{j}} \phi_{i \bar{\beta} k \bar{l}}+\phi_{\alpha \bar{j}} \phi_{k \bar{l}} \bar{j} \phi_{i \bar{\beta}} \\
& -\phi_{\alpha \bar{j} k} \phi_{j \bar{\beta} \bar{l}}-\phi_{\alpha \bar{j} \bar{l}} \phi_{j \bar{\beta} k} \\
= & \left(-\bar{\partial}\left(\partial \phi_{k \bar{t}} \phi^{\bar{t} s}\right) \phi_{s \bar{l}}\right)\left(\frac{\delta}{\delta z^{\alpha}}, \frac{\delta}{\delta \bar{z}^{\beta}}\right)-\left(\mu_{\alpha}\right)_{\bar{l}}^{j} \overline{\left(\mu_{\beta}\right)_{\bar{j}}^{s}} \phi_{k \bar{s}} \\
= & -R_{k \alpha \bar{\beta}}^{s} \phi_{s \bar{l}}-\left(\mu_{\alpha}\right)_{\bar{l}}^{j} \frac{\left(\mu_{\beta}\right)_{\bar{j}}^{s}}{\phi_{k \bar{s}},}
\end{aligned}
$$

where the last equality follows from (3.39). Consequently using (3.12) we get

$$
\begin{aligned}
\Delta c(\phi)_{\alpha \bar{\beta}} & =\phi^{\bar{l} k} \partial_{k} \partial_{\bar{l}} c(\phi)_{\alpha \bar{\beta}} \\
& =-R_{k \alpha \bar{\beta}}^{k}-\left(\mu_{\alpha}\right)_{\bar{l}}^{j} \overline{\left(\mu_{\beta}\right)_{\bar{j}}^{l}} \\
& =(\partial \bar{\partial} \log \operatorname{det} \phi)\left(\frac{\delta}{\delta z^{\alpha}}, \frac{\delta}{\delta \bar{z}^{\beta}}\right)-\left(\mu_{\alpha}\right)_{\bar{j}}^{i} \overline{\left(\mu_{\beta}\right)_{\bar{s}}^{t}} \phi^{\bar{j} s} \phi_{i \bar{t}},
\end{aligned}
$$

We perform the integration using Stoke's theorem on (3.43),

$$
\begin{aligned}
\int_{\mathcal{X} / M}( & \left.\sqrt{-1} R^{K \mathcal{X} / M}\right) \wedge \frac{\omega^{n}}{n !}=\int_{\mathcal{X} / M} \partial \bar{\partial} \log \operatorname{det} \phi\left(\frac{\delta}{\delta z^{\alpha}}, \frac{\delta}{\delta \bar{z}^{\beta}}\right) \frac{\omega^{n}}{n !} \sqrt{-1} d z^{\alpha} \wedge d \bar{z}^{\beta} \\
& +\int_{\mathcal{X} / M} \partial \bar{\partial} \log \operatorname{det} \phi\left(\frac{\delta}{\partial v^{i}}, \frac{\partial}{\partial \bar{v}^{j}}\right) \sqrt{-1} \delta v^{i} \wedge \delta \bar{v}^{j} \wedge c(\phi) \frac{\omega^{n-1}}{(n-1) !} \\
= & \int_{\mathcal{X} / M}\left(\left(\mu_{\alpha}\right)_{\bar{j}}^{i} \overline{\left(\mu_{\beta}\right)_{\bar{s}}^{t}} \phi^{\bar{j} s} \phi_{i \bar{t}} \sqrt{-1} d z^{\alpha} \wedge d \bar{z}^{\beta}-\rho c(\phi)\right) \frac{\omega^{n}}{n !} \\
= & -\int_{\mathcal{X} / M} \rho c(\phi) \frac{\omega^{n}}{n !}+\left\langle\mu_{\alpha}, \mu_{\beta}\right\rangle \sqrt{-1} d z^{\alpha} \wedge d \bar{z}^{\beta},
\end{aligned}
$$

which proves (3.43).

On the other hand we have also by (3.46) that

$$
\begin{aligned}
\left(\phi^{\bar{l} s} R_{s \bar{t}} \phi^{\bar{t} k}\right) \partial_{k} \partial_{\bar{l}} c(\phi)_{\alpha \bar{\beta}} & =\phi^{\bar{l} i} R_{i \bar{t}} \phi^{\bar{t} k}\left(-R_{k \alpha \bar{\beta}}^{s} \phi_{s \bar{l}}-\left(\mu_{\alpha}\right)_{\bar{l}}^{j} \overline{\left(\mu_{\beta}\right)_{\bar{j}}^{s}} \phi_{k \bar{s}}\right) \\
& =-R_{i \bar{t}} \phi^{\bar{t} k} R_{k \alpha \bar{\beta}}^{i}-\phi^{\overline{l i}} R_{i \bar{t}}\left(\mu_{\alpha}\right)_{\bar{l}}^{i} \overline{\left(\mu_{\beta}\right)_{\bar{j}}^{t}} \\
& =-R_{j \alpha \bar{\beta}}^{i} R_{i k \bar{l}}^{j} \phi^{k \bar{l}}-\left(\mu_{\alpha}\right)_{\bar{j}}^{i} \overline{\left(\mu_{\beta}\right)_{\bar{t}}^{s}} R_{i \bar{l}} \phi^{\bar{l} k} \phi^{\bar{j} t} \phi_{k \bar{s}} .
\end{aligned}
$$


By using Stoke's theorem again and noticing $\nabla_{k} \phi_{i}=0$, we have

$$
\begin{aligned}
& \int_{\mathcal{X} / M}(\Delta \rho) c(\phi) \frac{\omega^{n}}{n !}=\int_{\mathcal{X} / M} \phi^{\bar{l} s} \partial_{s} \partial_{\bar{l}}\left(R_{k \bar{t}} \phi^{\bar{t} k}\right) c(\phi) \frac{\omega^{n}}{n !} \\
& =\int_{\mathcal{X} / M} \phi^{\bar{l} s}\left(\nabla_{\bar{l}} \nabla_{s} R_{k \bar{t}}\right) \phi^{\bar{t} k} c(\phi) \frac{\omega^{n}}{n !} \\
& =\int_{\mathcal{X} / M} \phi^{\bar{l} s}\left(\nabla_{\bar{l}} \nabla_{k} R_{s \bar{t}}\right) \phi^{\bar{t} k} c(\phi) \frac{\omega^{n}}{n !} \\
& =\int_{\mathcal{X} / M} \phi^{\bar{l}_{s}} R_{s \bar{t}} \phi^{\bar{t} k} \nabla_{\bar{l}} \nabla_{k} c(\phi) \frac{\omega^{n}}{n !} \\
& =\int_{\mathcal{X} / M} \phi^{\bar{l} s} R_{s \bar{t}} \phi^{\bar{t} k} \partial_{k} \partial_{\bar{l}} c(\phi) \alpha \bar{\beta} \frac{\omega^{n}}{n !} \sqrt{-1} d z^{\alpha} \wedge d \bar{z}^{\beta} \\
& =\int_{\mathcal{X} / M}\left(-R_{j \alpha \bar{\beta}}^{i} R_{i k \bar{l}}^{j} \phi^{k \bar{l}}-\left(\mu_{\alpha}\right)_{\bar{j}}^{i} \overline{\left(\mu_{\beta}\right)_{\bar{t}}^{s}} R_{i \bar{l}} \phi^{\bar{l} k} \phi^{\bar{j} t} \phi_{k \bar{s}}\right) \frac{\omega^{n}}{n !} \sqrt{-1} d z^{\alpha} \wedge d \bar{z}^{\beta} \\
& =\left(-\int_{\mathcal{X} / M}\left(R_{j \alpha \bar{\beta}}^{i} R_{i k \bar{l}}^{j} \phi^{k \bar{l}}\right) \frac{\omega^{n}}{n !}-\left\langle\mu_{\alpha}, \mu_{\beta}\right\rangle_{R i c}\right) \sqrt{-1} d z^{\alpha} \wedge d \bar{z}^{\beta},
\end{aligned}
$$

which proves (3.44).

The first term in the RHS of (3.37) is, by (3.1) and Lemma 2.1,

$$
\begin{aligned}
\int_{\mathcal{X} / M} \frac{\omega^{n+1}}{(n+1) !} & =\int_{\mathcal{X} / M} \frac{\left(c(\phi)+\sqrt{-1} \phi_{i \bar{j}} \delta v^{i} \wedge \delta v^{j}\right)^{n+1}}{(n+1) !} \\
& =\int_{\mathcal{X} / M} \frac{(n+1) c(\phi)\left(\sqrt{-1} \phi_{i j} \delta v^{i} \wedge \delta v^{j}\right)^{n}}{(n+1) !} \\
& =\int_{\mathcal{X} / M} c(\phi) \frac{\omega^{n}}{n !} .
\end{aligned}
$$

We evaluate the Quillen curvature (3.37) at the vector $\zeta=\zeta^{\alpha} \frac{\partial}{\partial z^{\alpha}} \in T_{y} M$. It is, by (3.38), (3.41), (3.43), (3.44), (3.45) and (3.48),

$$
\begin{aligned}
& \left(\sqrt{-1} c_{1}(\lambda,\|\bullet\|)\right)(\zeta, \bar{\zeta}) \\
& =\frac{k^{n+1}}{(2 \pi)^{n+1}} \int_{\mathcal{X}_{y}}(-\sqrt{-1}) c(\phi)(\zeta, \bar{\zeta}) \frac{\omega^{n}}{n !}+\frac{k^{n}}{(2 \pi)^{n+1}} \int_{\mathcal{X}_{y}}\left(\frac{1}{2}|\mu|^{2}-\frac{\rho}{2}(-\sqrt{-1}) c(\phi)(\zeta, \bar{\zeta})\right) \frac{\omega^{n}}{n !} \\
& \quad+(-\sqrt{-1}) \frac{k^{n-1}}{(2 \pi)^{n+1}} \int_{\mathcal{X}_{y}} \frac{1}{8}\left(\sqrt{-1} R^{K \mathcal{X} / M}\right)^{2} \wedge \frac{\omega^{n-1}}{(n-1) !}(\zeta, \bar{\zeta}) \\
& \quad-\frac{k^{n-1}}{(2 \pi)^{n+1}} \frac{1}{24} \int_{\mathcal{X}_{y}}\left((-\sqrt{-1}) c(\phi)(\zeta, \bar{\zeta})\left(-2 \Delta \rho+|R i c|^{2}-|R|^{2}\right)\right) \frac{\omega^{n}}{n !} \\
& \quad+\frac{k^{n-1}}{(2 \pi)^{n+1}} \frac{1}{12}\left(\left\|\nabla^{\prime} \mu\right\|^{2}+\|\mu\|_{R i c}^{2}\right)+O\left(k^{n-2}\right) .
\end{aligned}
$$


Note that

$$
\begin{aligned}
& R^{K \mathcal{X} / M}=\partial \bar{\partial} \log \operatorname{det} \phi \\
& =(\partial \bar{\partial} \log \operatorname{det} \phi)\left(\frac{\delta}{\delta z^{\alpha}}, \frac{\delta}{\delta \bar{z}^{\beta}}\right) d z^{\alpha} \wedge d \bar{z}^{\beta}+(\partial \bar{\partial} \log \operatorname{det} \phi)\left(\frac{\delta}{\delta z^{\alpha}}, \frac{\partial}{\partial \bar{v}^{j}}\right) d z^{\alpha} \wedge \delta \bar{v}^{j} \\
& \quad+(\partial \bar{\partial} \log \operatorname{det} \phi)\left(\frac{\partial}{\partial v^{i}}, \frac{\delta}{\delta \bar{z}^{\beta}}\right) \delta v^{i} \wedge d \bar{z}^{\beta}+(\partial \bar{\partial} \log \operatorname{det} \phi)\left(\frac{\partial}{\partial v^{i}}, \frac{\partial}{\partial \bar{v}^{j}}\right) \delta v^{i} \wedge \delta \bar{v}^{j}
\end{aligned}
$$

and

$$
\bar{\partial}^{*} \mu_{\alpha}=(\partial \bar{\partial} \log \operatorname{det} \phi)\left(\frac{\delta}{\delta z^{\alpha}}, \frac{\partial}{\partial \bar{v}^{j}}\right) \phi^{\overline{j i}} \frac{\partial}{\partial v^{i}} .
$$

In fact, one can prove (3.51) in terms of normal coordinates, at a fixed point, one has

$$
\begin{aligned}
\bar{\partial}^{*} \mu_{\alpha} & =-\sqrt{-1}\left[\Lambda, \nabla^{\prime}\right]\left(\left(\mu_{\alpha}\right)_{\bar{j}}^{i} d \bar{v}^{j} \otimes \frac{\partial}{\partial v^{i}}\right) \\
& =-\phi^{s \bar{j}}\left(\partial_{s}\left(\mu_{\alpha}\right)_{\bar{j}}^{i}+\mu_{\bar{j}}^{k} \Gamma_{k s}^{i}\right) \frac{\partial}{\partial v^{i}} \\
& =\partial_{j} \partial_{\bar{j}}\left(\phi_{\alpha \bar{l}} \phi^{\bar{l} i}\right) \frac{\partial}{\partial v^{i}} \\
& =\left(\phi_{\alpha \bar{i} j \bar{j}}-\phi_{\alpha \bar{l}} \phi_{j} \bar{j} \bar{l}\right) \frac{\partial}{\partial v^{i}} \\
& =(\partial \bar{\partial} \log \operatorname{det} \phi)\left(\frac{\delta}{\delta z^{\alpha}}, \frac{\partial}{\partial \bar{v}^{j}}\right) \phi^{\bar{j} i} \frac{\partial}{\partial v^{i}} .
\end{aligned}
$$

By (3.50), (3.51), (3.42) and (3.47), the integral in the third term in the RHS of (3.49) can be computed as

$$
\begin{aligned}
& \int_{\mathcal{X} / M}\left(\sqrt{-1} R^{K \mathcal{X} / M}\right)^{2} \wedge \frac{\omega^{n-1}}{(n-1) !} \\
& =2 \int_{\mathcal{X} / M}(\partial \bar{\partial} \log \operatorname{det} \phi)\left(\frac{\delta}{\delta z^{\alpha}}, \frac{\delta}{\delta \bar{z}^{\beta}}\right)(\partial \bar{\partial} \log \operatorname{det} \phi)\left(\frac{\partial}{\partial v^{i}}, \frac{\partial}{\partial \bar{v}^{j}}\right) \sqrt{-1} \delta v^{i} \wedge \delta \bar{v}^{j} \frac{\omega^{n-1}}{(n-1) !} \sqrt{-1} d z^{\alpha} \wedge d \bar{z}^{\beta} \\
& -2 \int_{\mathcal{X} / M}(\partial \bar{\partial} \log \operatorname{det} \phi)\left(\frac{\delta}{\delta z^{\alpha}}, \frac{\partial}{\partial \bar{v}^{j}}\right)(\partial \bar{\partial} \log \operatorname{det} \phi)\left(\frac{\partial}{\partial v^{i}}, \frac{\delta}{\delta \bar{z}^{\beta}}\right) \sqrt{-1} \delta v^{i} \wedge \delta \bar{v}^{j} \frac{\omega^{n-1}}{(n-1) !} \sqrt{-1} d z^{\alpha} \wedge d \bar{z}^{\beta} \\
& +\int_{\mathcal{X} / M}(\partial \bar{\partial} \log \operatorname{det} \phi)\left(\frac{\partial}{\partial v^{i}}, \frac{\partial}{\partial \bar{v}^{j}}\right)(\partial \bar{\partial} \log \operatorname{det} \phi)\left(\frac{\partial}{\partial v^{k}}, \frac{\partial}{\partial \bar{v}^{l}}\right) \sqrt{-1} \delta v^{i} \wedge \delta \bar{v}^{j} \wedge \sqrt{-1} \delta v^{k} \wedge \delta \bar{v}^{l} c(\phi) \frac{\omega^{n-2}}{(n-2) !} \\
& =-2 \int_{\mathcal{X} / M} \rho\left(\Delta c(\phi)_{\alpha \bar{\beta}}+\left(\mu_{\alpha}\right)_{\bar{j}}^{i} \overline{\left(\mu_{\beta}\right)_{\bar{s}}^{t}} \phi^{\bar{j} s} \phi_{i \bar{t}}\right) \frac{\omega^{n}}{n !} \sqrt{-1} d z^{\alpha} \wedge d \bar{z}^{\beta} \\
& -2\left\langle\bar{\partial}^{*} \mu_{\alpha}, \bar{\partial}^{*} \mu_{\beta}\right\rangle \sqrt{-1} d z^{\alpha} \wedge d \bar{z}^{\beta}+\int_{\mathcal{X} / M}\left(\rho^{2}-|R i c|^{2}\right) c(\phi) \frac{\omega^{n}}{n !} .
\end{aligned}
$$

Evaluated at the vector $\zeta=\zeta^{\alpha} \frac{\partial}{\partial z^{\alpha}} \in T_{y} M$ it is, by Stoke's theorem,

$$
\begin{aligned}
(- & \sqrt{-1}) \int_{\mathcal{X} / M}\left(\sqrt{-1} R^{K \mathcal{X} / M}\right)^{2} \wedge \frac{\omega^{n-1}}{(n-1) !}(\zeta, \bar{\zeta}) \\
& =\int_{\mathcal{X}_{y}}\left((-\sqrt{-1}) c(\phi)(\zeta, \bar{\zeta})\left(-2 \Delta \rho+\rho^{2}-|R i c|^{2}\right)-2 \rho|\mu|^{2}\right) \frac{\omega^{n}}{n !}-2\left\|\bar{\partial}^{*} \mu\right\|^{2} .
\end{aligned}
$$

Substituting (3.52) into (3.49) we have proved 
Proposition 3.9 The curvature of the Quillen metric has the following expansion in $k$,

$$
\begin{aligned}
& \left(\sqrt{-1} c_{1}(\lambda,\|\bullet\|)\right)(\zeta, \bar{\zeta}) \\
& =\frac{k^{n+1}}{(2 \pi)^{n+1}} \int_{\mathcal{X}_{y}}(-\sqrt{-1}) c(\phi)(\zeta, \bar{\zeta}) \frac{\omega^{n}}{n !}+\frac{k^{n}}{(2 \pi)^{n+1}} \int_{\mathcal{X}_{y}}\left(\frac{1}{2}|\mu|^{2}-\frac{\rho}{2}(-\sqrt{-1}) c(\phi)(\zeta, \bar{\zeta})\right) \frac{\omega^{n}}{n !} \\
& \quad+\frac{k^{n-1}}{(2 \pi)^{n+1}} \int_{\mathcal{X}_{y}}\left((-\sqrt{-1}) c(\phi)(\zeta, \bar{\zeta})\left(-\frac{1}{6} \Delta \rho+\frac{1}{24}\left(|R|^{2}-4|R i c|^{2}+3 \rho^{2}\right)\right)-\frac{\rho}{4}|\mu|^{2}\right) \frac{\omega^{n}}{n !} \\
& \quad+\frac{k^{n-1}}{(2 \pi)^{n+1}}\left(\frac{1}{12}\|\mu\|_{R i c}^{2}+\frac{1}{12}\left\|\nabla^{\prime} \mu\right\|^{2}-\frac{1}{4}\left\|\bar{\partial}^{*} \mu\right\|^{2}\right)+O\left(k^{n-2}\right) .
\end{aligned}
$$

From above Proposition, we proved Theorem 1.2.

\subsection{An application}

In this subsection, we will prove Corollary 1.3.

For any positive integer $k$ write temporarily

$$
F=L^{k} \otimes K_{\mathcal{X} / M},
$$

where $K_{\mathcal{X} / M}$ is the relative canonical line bundle endowed with the following metric,

$$
(\operatorname{det} \phi)^{-1}:=\left(\operatorname{det}\left(\phi_{i} \bar{j}\right)\right)^{-1} .
$$

As in Sect. 2.3, the operator $D_{y}=\bar{\partial}_{y}+\bar{\partial}_{y}^{*}$ acts on $\oplus_{p \geq 0} A^{0, p}\left(\mathcal{X}_{y}, F\right)$. Take a small constant $b>0$ that is smaller than the all positive eigenvalues of $D_{y}$. Then

$$
\left.K_{y}^{b, p} \cong H^{p}\left(\mathcal{X}_{y}, F\right)=H^{p}\left(\mathcal{X}_{y}, K_{\mathcal{X}_{y}}\right) \otimes L^{k}\right) .
$$

By Kodaira vanishing theorem,

$$
K_{y}^{b, 0} \cong H^{0}\left(\mathcal{X}_{y}, L^{k} \otimes K_{\mathcal{X}_{y}}\right) \cong \pi_{*}\left(L^{k} \otimes K_{\mathcal{X} / M}\right)_{y} \quad K_{y}^{b, p}=0, \quad \text { for } \quad p \geq 1 .
$$

So

$$
\lambda^{b}=\left(\operatorname{det} \pi_{*}\left(L^{k} \otimes K_{\mathcal{X}_{y}}\right)\right)^{-1} .
$$

By (2.3) and (3.54), we have

$$
|u|^{2} e^{-k \phi}=(\sqrt{-1})^{2}\left|u^{\prime}\right|^{2} e^{-k \phi} d v \wedge d \bar{v}=\left|u^{\prime}\right|^{2} e^{-k \phi}(\operatorname{det} \phi)^{-1} \frac{\omega^{n}}{n !}=|u|_{L^{2}}^{2} \frac{\omega^{n}}{n !},
$$

that is, the $L^{2}$-metric $\|\bullet\|_{k}$ on $\pi_{*}\left(L^{k} \otimes K_{\mathcal{X} / M}\right)$ given by (2.3) coincides with the standard $L^{2}$ metric on $\pi_{*}\left(L^{k} \otimes K_{\mathcal{X} / M}\right)$ induced by $\left(\mathcal{X}_{y},\left.\omega\right|_{y}\right),\left(K_{\mathcal{X}_{y}},(\operatorname{det} \phi)^{-1}\right)$ and $\left(L, e^{-\phi}\right)$. Therefore, the $L^{2}$-metric $\left(|\bullet|^{b}\right)^{2}$ is dual to the determinant of the metric $\|\bullet\|^{2}$. By (2.16), we have

$$
\left(\|\bullet\|^{b}\right)^{2}=\left(|\bullet|^{b}\right)^{2}\left(\tau_{k}\left(\bar{\partial}^{(b,+\infty)}\right)\right)^{2}=\left(\operatorname{det}\|\bullet\|_{k}^{2}\right)^{*}\left(\tau_{k}(\bar{\partial})\right)^{2},
$$

for $b>0$ small enough, where $\tau_{k}(\bar{\partial})=\tau_{k}\left(\bar{\partial}^{(b,+\infty)}\right)$ is the analytic torsion associated with $(\mathcal{X}, \omega=\sqrt{-1} \partial \bar{\partial} \phi))$ and $\left(L^{k}, e^{-k \phi}\right)$. Therefore,

$$
\frac{\sqrt{-1}}{2 \pi} \partial \bar{\partial} \log \left(\tau_{k}(\bar{\partial})\right)^{2}=-c_{1}\left(\lambda,\|\bullet\|_{Q}\right)-c_{1}\left(E^{k},\|\bullet\|_{k}\right) .
$$




\section{Proof of Corollary 1.3}

Substituting (3.36) and (3.53) into (3.58), we obtain

$$
\begin{aligned}
\frac{1}{2 \pi} \partial \bar{\partial} \log \left(\tau_{k}(\bar{\partial})\right)^{2}(\zeta, \bar{\zeta}) & =(-\sqrt{-1})\left(-c_{1}\left(\lambda,\|\bullet\|_{Q}\right)-c_{1}\left(E^{k},\|\bullet\|_{k}\right)\right)(\zeta, \bar{\zeta}) \\
& =o\left(k^{n-1}\right) .
\end{aligned}
$$

Therefore,

$$
\partial \bar{\partial} \log \tau_{k}(\bar{\partial})=o\left(k^{n-1}\right) .
$$

Acknowledgements We would like to thank Professor Bo Berndtsson for patiently answering us several questions and for inspiring discussions, and Professor Xiaonan Ma for drawing our attention to the preprint [19]. We are also grateful to Siarhei Finski who explained us carefully his results in a written communication.

Funding Open access funding provided by Chalmers University of Technology. Funding was provided by Vetenskapsrådet (Grant No. 11253578). Xueyuan Wan is partially supported by Scientific Research Foundation of Chongqing University of Technology.

Open Access This article is licensed under a Creative Commons Attribution 4.0 International License, which permits use, sharing, adaptation, distribution and reproduction in any medium or format, as long as you give appropriate credit to the original author(s) and the source, provide a link to the Creative Commons licence, and indicate if changes were made. The images or other third party material in this article are included in the article's Creative Commons licence, unless indicated otherwise in a credit line to the material. If material is not included in the article's Creative Commons licence and your intended use is not permitted by statutory regulation or exceeds the permitted use, you will need to obtain permission directly from the copyright holder. To view a copy of this licence, visit http://creativecommons.org/licenses/by/4.0/.

\section{References}

1. Andersen, J.E.: Hitchin's connection, Toeplitz operators and symmetry invariant deformation quantization. Quantum Topol. 3(3-4), 293-325 (2012)

2. Andersen, J.E., Gammelgaard, N.L., Lauridsen, M.R.: Hitchin's connection in metaplectic quantization. Quantum Topol. 3(3-4), 327-357 (2012)

3. Andersen, J.E., Rasmussen, K.: A Hitchin connection for a large class of families of Kähler structures. In: Andersen, J.E., Dancer, A., Garcia-Prada, O. (eds.) Geometry and Physics, Festschrift in Honour of Nigel Hitchin, vol. 1, pp. 135-162. Oxford University Press, Oxford (2018)

4. Berman, R., Berndtsson, B., Sjöstrand, J.: A direct approach to Bergman kernel asymptotics for positive line bundle. Ark. Mat. 46, 197-217 (2008)

5. Berman, R.: Tunneling, the Quillen metric and analytic torsion for high powers of a holomorphic line bundles. Contemp. Math. 630, 15-31 (2014)

6. Berndtsson, B.: Curvature of vector bundles associated to holomorphic fibrations. Ann. Math. 169, 531560 (2009)

7. Berndtsson, B.: Positivity of direct image bundles and convexity on the space of Kähler metrics. J. Differ. Geom. 81(3), 457-482 (2009)

8. Berndtsson, B.: Strict and non strict positivity of direct image bundles. Math. Z. 269, 1201-1218 (2011)

9. Berline, N., Getzler, E., Vergne, M.: Heat Kernels and Dirac Operators. Springer, Berlin (1996)

10. Bismut, J.-M., Vasserot, E.: The asymptotics of the Ray-Singer torsion associated with high powers of a positive line bundle. Commun. Math. Phys. 125, 355-367 (1989)

11. Bismut, J.-M., Gillet, H., Soulé, C.: Analytic torsion and holomorphic determinant bundles I. Bott-Chern forms and analytic torison. Commun. Math. Phys. 115, 49-78 (1988)

12. Bismut, J.-M., Gillet, H., Soulé, C.: Analytic torsion and holomorphic determinant bundles II. Direct images and Bott-Chern forms. Commun. Math. Phys. 115, 79-126 (1988)

13. Bismut, J.-M., Gillet, H., Soulé, C.: Analytic torsion and holomorphic determinant bundles III. Quillen metrics on holomorphic determinants. Commun. Math. Phys. 115, 301-351 (1988) 
14. Catlin, D.: The Bergman kernel and a theorem of Tian. In: Analysis and Geometry in Several Complex Variables, pp. 1-23. Katata; 1997

15. Demailly, J.-P.: Complex analytic and differential geometry (2012). https://www-fourier.ujf-grenoble.fr/ $\sim$ demailly/manuscripts/agbook.pdf

16. Dai, X., Liu, K., Ma, X.: On the asymptotic expansion of Bergman kernel. J. Differ. Geom. 72, 1-41 (2006)

17. Feng, H., Liu, K., Wan, X.: Geodesic-Einstein metrics and nonlinear stabilities. Trans. Am. Math. Soc. 371(11), 8029-8049 (2019)

18. Fedosova, K., Rowlett, J., Zhang, G.: The second variation of Selberg zeta functions and plurisubharmonicity. Ann. Glob. Anal. Geom. 57(1), 23-60 (2020)

19. Finski, S.: On the full asymptotic of analytic torsion. J. Funct. Anal. 275(12), 3457-3503 (2018)

20. Lu, Z.: On the lower order terms of the asymptotic expansion of Tian-Yau-Zelditch. Am. J. Math. 122(2), 235-273 (2000)

21. Ma, X., Marinescu, G.: Holomorphic Morse Inequalities and Bergman Kernels. Birkhäuser, Basel (2006)

22. Ma, X., Zhang, W.: Superconnection and family Bergman kernels. C. R. Acad. Sci. Pairs Ser. I(344), 41-44 (2007)

23. Ray, D.B., Singer, I.M.: Analytic torsion for complex manifolds. Ann. Math. 98(1), 154-177 (1973)

24. Sun, X.: Deformation of canonical metric I. Asian J. Math. 16, 141-156 (2012)

25. Tian, G.: On a set of polarized Kähler metrics on algebraic manifolds. J. Differ. Geom. 32, 99-130 (1990)

26. Wang, L.: Bergman kernel and stability of holomorphic vector bundles with sections, MIT PhD. Dissertation, 85 pages (2003)

27. Xu, H.: A closed formula for the asymptotic expansion of the Bergman kernel. Commun. Math. Phys. 314, 555-585 (2012)

28. Zelditch, S.: Szegö kernel and a theorem of Tian. Int. Math. Res. Not. 6, 317-331 (1998)

Publisher's Note Springer Nature remains neutral with regard to jurisdictional claims in published maps and institutional affiliations. 